\title{
Performance and Thermal Tradeoffs for Energy-Efficient Monolithic 3D Network-on-Chip
}

\author{
DONGJIN LEE, SOURAV DAS, JANARDHAN RAO DOPPA, and \\ PARTHA PRATIM PANDE, Washington State University \\ KRISHNENDU CHAKRABARTY, Duke University
}

\begin{abstract}
Three-dimensional (3D) integration enables the design of high-performance and energy-efficient network on chip (NoC) architectures as communication backbones for manycore chips. To exploit the benefits of the vertical dimension of 3D integration, through-silicon-via (TSV) has been predominantly used in state-of-theart manycore chip design. However, for TSV-based systems, high power density and the resultant thermal hotspot remain major concerns from the perspectives of chip functionality and overall reliability. The power consumption and thermal profiles of 3D NoCs can be improved by incorporating a Voltage-Frequency-Island (VFI)-based power management strategy. However, due to inherent thermal constraints of a TSV-based 3D system, we are unable to fully exploit the benefits offered by the power management methodology. In this context, emergence of monolithic 3D (M3D) integration has opened up new possibility of designing ultra-lowpower and high-performance circuits and systems. The smaller dimensions of the inter-layer dielectric (ILD) and monolithic inter-tier vias (MIVs) offer high-density integration, flexibility of partitioning logic blocks across multiple tiers, and significant reduction of total wire-length. In this work, we present the first-ever study of the performance-thermal tradeoffs for energy efficient monolithic 3D manycore chips. In particular, we present a comparative performance evaluation of M3D NoCs with respect to their conventional TSVbased counterparts. We demonstrate that the proposed M3D-based NoC architecture incorporating VFI-based power management achieves a maximum of $29.4 \%$ lower energy-delay-product (EDP) compared to the TSVbased designs for a large set of benchmarks. We also demonstrate that the M3D-based NoC shows up to $29.1 \%$ lower maximum temperature than the TSV-based counterpart for these benchmarks.
\end{abstract}

CCS Concepts: $\bullet$ Networks $\rightarrow$ Network on chip; $\bullet$ Computer systems organization $\rightarrow$ Interconnection architectures; $\bullet$ Hardware $\rightarrow$ 3D integrated circuits; Chip-level power issues;

Additional Key Words and Phrases: 3D NoC, monolithic 3D, VFI power management, thermal hotspots

\section{ACM Reference format:}

Dongjin Lee, Sourav Das, Janardhan Rao Doppa, Partha Pratim Pande, and Krishnendu Chakrabarty. 2018. Performance and Thermal Tradeoffs for Energy-Efficient Monolithic 3D Network-on-Chip. ACM Trans. Des. Autom. Electron. Syst. 23, 5, Article 60 (August 2018), 25 pages.

http://dx.doi.org/10.1145/3223046

This work was supported by the US National Science Foundation (NSF) grants CNS-1564014 and CCF 1514269 and USA Army Research Office grant W911NF-17-1-0485.

Author's addresses: D. Lee, S. Das, J. R. Doppa, and P. P. Pande, School of Electrical Engineering and Computer Science, Washington State University, Box 642752, Pullman, Washington 99164; emails: \{dongjin.lee, sourav.das, jana.doppa, pande\}@wsu.edu; K. Chakrabarty, Department of Electrical and Computer Engineering, Duke University, Box 90291,130 Hudson Hall, Durham, NC 27708; email: krishnendu.chakrabarty@duke.edu.

Permission to make digital or hard copies of all or part of this work for personal or classroom use is granted without fee provided that copies are not made or distributed for profit or commercial advantage and that copies bear this notice and the full citation on the first page. Copyrights for components of this work owned by others than ACM must be honored. Abstracting with credit is permitted. To copy otherwise, or republish, to post on servers or to redistribute to lists, requires prior specific permission and/or a fee. Request permissions from permissions@acm.org.

(C) 2018 ACM 1084-4309/2018/08-ART60 $\$ 15.00$

http://dx.doi.org/10.1145/3223046 


\section{INTRODUCTION}

Three-dimensional Network-on-Chip (3D NoC) has emerged as a promising approach to design high-performance and energy-efficient manycore chips. It combines the benefits of high integration density and energy efficiency of 3D integration and scalability of network-on-chip-based connectivity to offer unprecedented performance gain. Through-silicon-via (TSV) is the most popular means to establish vertical connectivity between the planar stacked layers. Through the use of multiple planar layers and TSV-based vertical links, the 3D NoC enables smaller overall footprints with significant performance improvement over conventional 2D counterparts. However, there are several challenges associated with the TSV-based 3D integration. First, the fabrication challenges lead to low yield rates and high production cost of the TSVs. Second, while significant progress has been achieved to fabricate TSVs with small dimensions, however, according to the latest ITRS report, the diameter and length of the TSV are still order of magnitude larger than upper-level metals and standard metal pitch (ITRS 2015). Moreover, TSV-based 3D NoC requires the minimum keep-out-zone (KOZ) to prevent the impact of TSV-induced stress and coupling noise, which introduces additional area overhead. In addition, the parasitic and cross-coupling capacitances present among the TSVs of a bundle cause additional power consumption and degrade the reliability of the TSV-enabled links (Yaghini et al. 2015).

Another major challenge associated with TSV-based 3D NoC architectures is the high-power density and the resultant thermal hotspots leading to reliability concerns over time. Creation of thermal hotspots adversely affects the performance of the manycore chip, compromising reliability and signal integrity and eventually giving rise to possible higher cooling cost (Lee and Chakrabarty 2009; Coskun et al. 2009).

One of the possible ways to address the power density issue of a 3D NoC is to incorporate suitable power management strategies. In this context, Voltage-Frequency Island (VFI)-based power management is a well-known methodology to lower the overall energy consumption within a given performance constraint (Ogras et al. 2009; Jang and Pan 2011). Hence, it has been considered as an effective way to handle the thermal hotspots of conventional 2D as well as 3D manycore chips (Huang et al. 2004; Coskun et al. 2007; Juan et al. 2014). However, even after incorporating VFI-based power management, it has been found that the relatively higher power consuming VFIislands are generally placed near the heat sink to reduce the temperature hotspots (Das et al. 2004; Kumar et al. 2014a). The resultant restriction on the placement of the VFI-islands often leads to a manycore system configuration where highly communicating cores may be placed far apart. This results in increased overall hop count and introduces additional overall performance penalty.

Recently, monolithic 3D (M3D) integration has been proposed as a breakthrough technology to achieve significant performance improvement and energy efficiency. It opens up the possibility of designing cores and associated network routers using multiple tiers by utilizing monolithic intertier vias (MIVs) and hence, reduces the effective wire length (Lee et al. 2013). Compared to TSVbased 3D ICs, M3D offers the "true" benefits of vertical dimension for system integration: the size of a MIV used in M3D is over 100x smaller than a TSV (Batude et al. 2014; Nayak et al. 2015). To explain the TSV- and M3D-based integrations, Figure 1(a) shows an example of 3D design with these two technologies, while Figure 1(b) illustrates the comparison of physical dimensions between the TSVs and MIVs. This dramatic reduction in via size of the MIVs and the resulting increase in density of the M3D-integration opens up numerous opportunities for design optimizations in 3D NoCs. In addition, for M3D-enabled architecture, due to the presence of extremely thin inter-layer dielectric (ILD) layers in between planar tiers, and very small parasitic capacitance for MIV-based interconnects, M3D-enabled NoC offers significantly higher integration density than TSV-based configurations (Lee et al. 2013; Turkyilmaz et al. 2014). In addition, M3D-integration can overcome the drawback of TSV-based 3D IC. Unlike the TSVs, additional space for keep-out-zone (KOZ) is not 


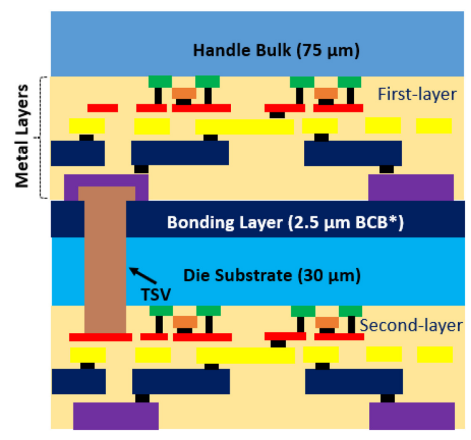

TSV-based 3D IC

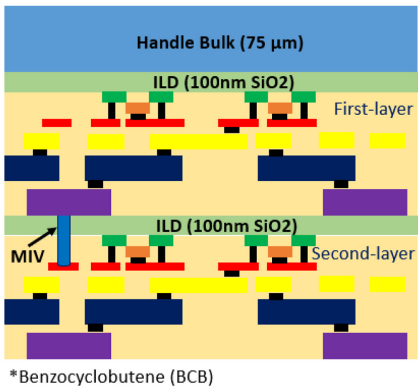

Monolithic 3D IC

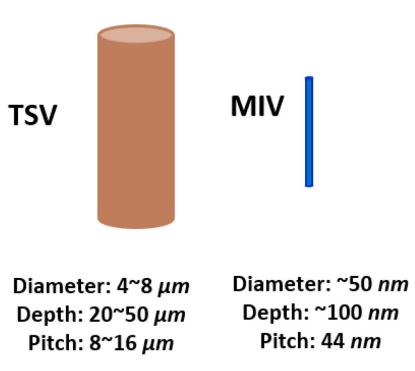

(b)

Fig. 1. (a) Illustration of Monolithic 3D (M3D) and TSV-based 3D structure. While two thick layers, i.e., bonding layer and die substrate are present between the tiers for TSV-based integration, however, for M3Denabled architecture, only thin inter-layer dielectric (ILD) layer is present. The figure is redrawn from Samal et al. (2014). (b) Comparison of physical dimensions of TSV and MIV vias. (ITRS 2015).

required for MIVs. The MIVs are similar to conventional vias in dimensions and parasitic values. Hence, active circuitries can be placed in vicinity of MIVs, resulting in significant improvement over TSV-based integrations.

The physical dimensions of TSVs are significantly large compared to MIVs used in M3D-integrations. For example, the diameter of TSVs for today's technology nodes is $2 \sim 5 \mu \mathrm{m}$ and if we consider a keep-out-zone of $2.5 \mu \mathrm{m}$ for each TSV, then the total silicon area for a single TSV is on the order of $100 \mu \mathrm{m}^{2}$ (Liu and Lim 2012). This is equivalent to $5 \mathrm{x}$ the standard cell-area in $45 \mathrm{~nm}$ technology. The parasitic capacitance value for the TSV is also large. For TSVs with diameters of $2 \mu \mathrm{m}$ and $5 \mu \mathrm{m}$, the capacitance values are $3 \mathrm{fF}$ and $20 \mathrm{fF}$, respectively (Liu and Lim 2012; Samal et al. 2016). In this scenario, the maximum number of TSVs that can be used in a $1 \mathrm{~mm} \times 1 \mathrm{~mm}$ die is limited to 3000 (considering that $30 \%$ of the area is allocated for TSVs) (Liu and Lim 2012). In addition, if the TSV diameter is reduced, then the yield is reduced for TSV-based ICs (Souriau et al. 2012). For MIVs, the diameter is comparable to that of regular $\mathrm{Cu}$ vias and it is in the order of 50 $\mathrm{nm}$ (Samal et al. 2016). The parasitic capacitance of MIV is $0.2 \mathrm{fF}$ (Samal et al. 2016). This enables high-density integration for M3D. For a simple LDPC benchmark, the number of MIVs in a single chip can be more than 80k (Nayak et al. 2015) while the number can be more than 30 million for $1 \mathrm{~mm} \times 1 \mathrm{~mm}$ chip if we consider the same 30\% area of the chip (Liu and Lim 2012). Moreover, the TSV manufacturing costs for 3D integration is high due to limited yield rate. For example, an 83\% yield rate with state-of-the-art manufacturing for an 8-inch wafer is considered as the bestachieved rate for TSV-based ICs (Lau 2010; Watanabe et al. 2016, 2017). On the other hand, the direct wafer-bonding technique in M3D does not face such difficulties, and hence, achieves high yield and low cost (Batude et al. 2012; Uhrmann et al. 2014). Consequently, the M3D-based design ensures better performance per power per cost (PPC) when the number of MIVs increase in the chip. The PPC benefit of M3D can be $25 \%$ higher compared to the mini-sized TSV-based $(2 \mu \mathrm{m}$ diameter) designs (Nayak et al. 2015).

From the thermal profile perspective, one of the advantages of M3D integration is that in an M3D-enabled architecture, the inter-layer dielectric (ILD) used between planar tiers is very thin. In addition, there is no bonding layer (which has poor thermal conductivity) present between adjacent tiers, which facilitate smooth thermal flow across the chip (Samal et al. 2014; Santos 
et al. 2016). In contrast, in a TSV-based design, the thick silicon substrate and the presence of the bonding layer obstruct the heat flow from the source of power dissipation to the heat sink and aggravate temperature profiles of the planar layers in a 3D design (Samal et al. 2014).

In this work, we propose a detailed design methodology of a performance- and thermal-efficient M3D-enabled NoC architecture incorporating VFI-based power management. This is the first-ever study that analyzes the performance of an M3D-enabled NoC. The proposed approach explores the possibility of designing an efficient 3D NoC enabled by true multi-tier routers to exploit the full benefit from the monolithic structure. We use a small-world network 3D NoC architecture (3D SWNoC) that achieves lower latency and energy dissipation compared to other state-of-theart counterparts (Das et al. 2015). We address the full system power and thermal profiles of a manycore chip with the TSV- and M3D-enabled 3D SWNoC as the communication backbone. We present a holistic design flow to address performance and thermal tradeoffs of 3D SWNoC-based manycore design.

The rest of this article is organized as follows: Section 2 discusses the previous works done in the field of M3D, and NoC design and optimization. The M3D-enabled NoC with VFI power management is elaborated in Section 3. The details of the optimization methodology for VFI-enabled performance and thermal efficient NoC architectures are described in Section 4. We discuss an exhaustive performance evaluation in Section 5. Finally, Section 6 concludes this article by highlighting the salient features of this work.

\section{RELATED WORK}

3D NoC has emerged as one of the compelling solutions to design high performance and energyefficient communication infrastructure for manycore chips. The natural extension of 2D planar architectures was the simple and regular 3D MESH-based NoC, which has been investigated in many existing works (Kim et al. 2007; Marcon et al. 2014). To exploit the advantages of TSV-based 3D integration, several works have addressed the synthesis of application-specific NoC architectures. The Sunfloor 3D was proposed for developing application-specific 3D NoCs (Seiculescu et al. 2009). The design and synthesis of application-specific 3D NoC architectures were also investigated in several works (Zhou et al. 2010; Murali et al. 2009). Later, a more general-purpose 3D $\mathrm{NoC}$ was proposed (Xu et al. 2009) using an Integer Linear Programming (ILP)-based algorithm to insert long-range links to develop low-diameter and low-radix architecture. The main focus of all of the above-mentioned TSV-based 3D NoC architectures is the performance improvement without considering the thermal concerns of the system. Consequently, the architecture faces two major implications. First, large temperature gradients are observed across the chip resulting in non-uniform slow-down of the transistor speed, accelerated negative body-biased temperature instability (NBTI) issues, overall performance degradation of the chip, and increased reliability concerns (Schroder and Babcock 2003; Schroder 2007). Second, and more importantly, in most of the cases, thermal hotspots are formed, which worsens the reliability for certain areas of the chip.

Increasing the power density and the resultant thermal hotspots are well-known limitations of 3D integration. There is a significant body of work that has dealt with various thermal issues in TSV-based 3D ICs. Insertion of microfluidic channel (MFC) is one of the most popular approaches to mitigate the thermal hotspot problem in a 3D IC (Sabry et al. 2013; Shi et al. 2011; Dang et al. 2010). In addition, redundant TSV has also been utilized as the conducting channel between the planar dies of 3D ICs for dissipating the heat. In this context, a clustering-based redundant TSV insertion technique was proposed to remove heat in 3D ICs (Oh et al. 2012). However, insertion of extra TSV is costly, and it also affects the performance of neighboring TSVs. To spread the heat widely and effectively for the whole system, cooling architectures using thermal sidewalls, inter-chip plates, and a bottom plate (known as the thermal-SIB architecture) are proposed (Furumi 
et al. 2017). Consequently, exploring alternative vertical integration technology has been identified as one of the major targets for next generation of 3D ICs and systems.

In order to improve the performance of 3D-integrated systems, several semiconductor companies (e.g., GLOBALFOUNDRIES and Qualcomm in collaboration with CEA-LETI) are exploring M3D as a means for overcoming the pitch and alignment limitations of TSVs. In order to ensure that M3D integration is primed for use when industry is ready to adopt it, researchers have developed a low-temperature process to fabricate high-performance top transistor layers without degrading the performance of bottom transistor layers (Ishihara et al. 2012). Design techniques to reduce interconnect length, critical path delay, and die area were presented in Bobba et al. (2011) and Samal et al. (2016). An M3D IC can be partitioned at the core-, block-, gate- and transistor-level. Gate-level partitioning appears to be the most promising (Panth et al. 2014) and it is especially relevant to 3D NoC design. Therefore, significant research efforts are being directed towards the development of design tools for gate-level-partitioned M3D ICs (Lee et al. 2013; Bobba et al. 2011). In addition, recently the thermal profiles of M3D- and TSV-based 3D IC have been explored considering their material and structural difference (Samal et al. 2014; Santos et al. 2016). In TSV-based 3D ICs, the die thickness and the distance between the active devices in adjacent layers ranges from $20 \mu \mathrm{m}$ to $80 \mu \mathrm{m}$ (Souriau et al. 2012). In addition, a bonding layer (Benzocyclobutene - BCB) is used in between dies for TSV-based integration. The thermal conductivity of this BCB-based bonding layer is poor $(0.29 \mathrm{~W} / \mathrm{m}-\mathrm{K})$ (Samal et al. 2014), hence, heat flow is impeded. On the other hand, no bonding layer is used in M3D-based integration and a thin layer of $\mathrm{SiO}_{2} \operatorname{ILD}(100 \sim 300 \mathrm{~nm})$ is present between the adjacent tiers. The thermal conductivity $\mathrm{SiO}_{2}\left(\mathrm{SiO}_{2}\right.$ has the conductivity of $1.38 \mathrm{~W} / \mathrm{m}-\mathrm{K}$ ) is four times higher than that of BCB (Samal et al. 2014). Hence, it facilitates better thermal flow for M3D designs. Therefore, the M3D design leads to lower maximum temperature and fewer hotspots than TSV-based design.

In this context, we advocate exploiting M3D-integration to design efficient small-world-based NoC (SWNoC) architecture with VFI-based power management. We address the full system power and thermal profiles of the M3D manycore chip with the 3D SWNoC as the communication backbone. We compare the performance and thermal profiles of TSV- and M3D-enabled SWNoC architectures by incorporating VFI-based power management. Also, we present a holistic design flow to address the performance and thermal tradeoffs of an energy-efficient M3D manycore design.

\section{DESIGN OF M3D NOC ARCHITECTURE WITH VFI}

In this section, we formally introduce the M3D-enabled NoC design space. Since a TSV-based 3D SWNoC outperforms other regular and irregular NoC architectures in terms of network latency and energy efficiency (Das et al. 2015), we consider it as the testbed in this article to demonstrate the benefits of M3D-integration. Subsequently, to improve the energy efficiency, we present the design methodology to incorporate VFI-based power management in the adopted 3D NoC architecture.

\subsection{NoC Design with M3D Integration}

In an NoC, the network routers form the communication backbone. The total network energy consumption consists of the link and router energies. In order to reduce the energy consumption and network latency of the 3D NoC, the benefits of M3D integration can be exploited. More specifically, the routers can be designed to extend over multiple tiers. The benefits of extending the routers over multiple tiers are three-fold. First, the intra-router wire length and the energy consumption are reduced. Second, the inter-router hop count of the network reduces as the routers extended over multiple tiers can communicate directly with other routers without going through different tiers. By designing the routers over two tiers in M3D-integration for a 3D MESH NoC, we can save 30\% in footprint, and $16 \%$ in energy per message compared to their TSV counterpart (Das et al. 2017). 


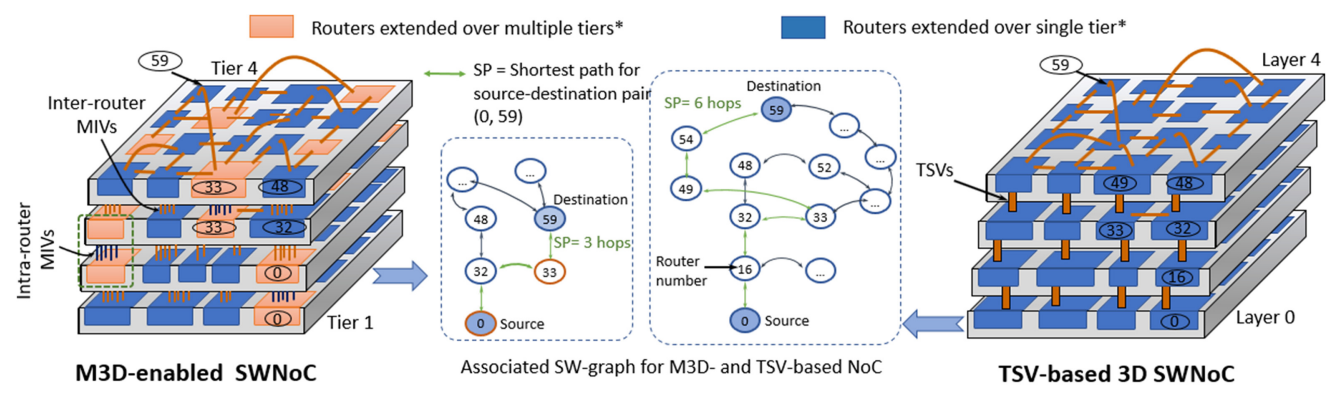

* For simplicity, only the router tiles and the network connectivity are shown (associated cores connected to routers are not shown). For M3Denabled architecture, routers, those are extended over multiple-tiers, are colored with (light) orange while the single-tier routers are shown in blue.

Fig. 2. Illustration of a small-world (SW) network-enabled 64-node 3D NoC architecture with M3D- and TSV-integrations.

Third, the TSVs that consume high energy are replaced with energy-efficient MIVs. While the energy consumption of MIVs is comparable to that of planar metal wires, the associated energy per bit for the TSVs are higher. Depending on the implementation methodology and technology parameters, the values of the typical TSV energy per bit are $4 \sim 5$ times more than that of metal wires (Lee et al. 2013; Samal et al. 2016).

To illustrate the benefits of an M3D SWNoC over the TSV-enabled counterpart, Figure 2 shows a conceptual view of TSV- and M3D-based 3D SWNoCs. As seen in this figure, in the M3D SWNoC, in addition to the single-tier routers (blue), some routers are extended over multiple tiers (orange). In contrast, for a TSV-based design, all the routers are placed on a single planar layer (blue). To explain how this helps to improve the performance of 3D NoC, we consider the inter-node communications for the source-destination pair of $(0,59)$ as an example. The associated SW-graph connectivity is shown in the middle part of Figure 2. The path highlighted with green indicates the shortest available path for this particular pair. The shortest-path (SP) lengths for M3D- and TSV-based SWNoC are calculated to be 3 and 6 hops respectively, which shows significant reduction in hop count for the M3D configuration. Additionally, we get improvements in energy as MIVs are more energy efficient compared to TSVs. In the next section, we explore how to exploit these benefits to design an energy-efficient 3D NoC architecture.

\subsection{Small-World Network-Enabled M3D NoCs}

A small-world (SW) network lies between a regular locally interconnected MESH network and a completely random Erdös-Rényi topology. The average shortest path length of a SW graph is bounded by a polynomial in $\log (N)$, where $N$ refers to the number of nodes (Nguyen and Martel 2005; Petermann and Rios 2005). In an SWNoC architecture, the achievable performance largely depends on how efficiently the network is optimized for a given set of performance metrics (Ogras and Marculescu 2006; Das et al. 2015). Here, the optimization of the network refers to the placement of the cores, routers, and associated horizontal and vertical links to ensure the best achievable performance.

For the TSV-based architectures, vertical links only connect routers from adjacent planar layers in point-to-point (P2P) manner. To exploit the benefits of small-world network for the TSV-based NoCs, the placement of the horizontal links for a given set of routers are optimized to maximize the performance gain. In the context of M3D-based design, the logic blocks, and hence the routers, are extended over multiple tiers, and their respective placements become completely irregular in the design space. Unlike the TSV-based designs, the vertical links of M3D-integration not only 
implement the fixed P2P connection, but also enable the intra-router connectivity spanning over multiple tiers. Hence, to ensure high performance, it is required to explore which M3D-enabled routers are to be extended over multiple tiers and how many of them to be designed with single tier. Consequently, the placement of both routers and links (horizontal and vertical) are crucial for achieving high performance and energy efficiency for M3D-enabled NoCs.

\subsection{Problem Formulation}

The NoC design space consists of a set of cores, $C=\left\{C_{1}, C_{2}, \ldots, C_{M}\right\}$, where $M$ refers to the total number of cores. Each core is connected to a nearby router and the connectivity among the routers forms a small-world network. In addition, the whole design space consists of $P$ number of tiers, and the vertical communications are enabled via MIVs. The link distribution of the network is denoted by $L$, where $L=\left\{l_{1}, l_{2}, \ldots, l_{t}\right\}$ and $l_{i}$ denotes the number of links having a particular link length $i$. Here, the parameter $t$ indicates the maximum link-length present in the network.

The performance of the M3D-enabled SWNoC depends on the effectiveness of the joint placement of the routers and links in the NoC design space (Das et al. 2017). To optimize the link and router placements, we define a unified cost function called the communication path length and aim to achieve the lowest value of the communication cost of the network.

The unified cost function, $O$, that combines all the relevant performance metrics (Das et al. 2015) is defined as:

$$
O=\sum_{i=1}^{N} \sum_{j=1}^{N}\left(r \cdot h_{i j}+l_{i j}\right) \cdot f_{i j} \quad(i \neq j)
$$

where $N, h_{i j}, l_{i j}$, and $f_{i j}$ denote the total number of nodes, the hop count, the minimum link length, and the frequency of interaction between the $i$ th and $j$ th nodes, respectively. The parameter $r$ refers to the routing stages including the virtual channel allocation, input- and output-port arbitration, path allocation inside a router. Optimizing the cost function, $O$, ensures lower average hop count, (near-) optimal link and router placements to ensure reduced resource utilization. For TSV-based NoC architectures, while the placement of the horizontal and vertical link ensures exploration of the (near-) optimal configuration; however, for M3D-enabled SWNoC, in addition to these, extension of routers to multiple tiers also significantly affects the value of the cost function. As a result, the router placement is an additional challenge for an M3D-enabled NoC architecture. Subsequently, by minimizing the communication path length, both the router and link energy consumptions are reduced, leading to improvement in the overall NoC performance.

\subsection{Network Constraints}

To design the M3D SWNoC architecture, we incorporate the physical constraints imposed by the M3D technology. For the TSV-based designs, we could only allow the placement of vertical links point-to-point (regularly) between routers placed at different planar layers. In addition, the logic blocks needed to design the routers are constrained to be placed in a single planar die. On the other hand, for M3D-based integration, routers are extended over multiple tiers. However, even with the current state-of-the-art fabrication technology, if any logic block is extended over more than two tiers, the performance varies among different tiers due to a mismatch in transistor speed and interconnect-material (e.g., Copper $(\mathrm{Cu})$ and Tungsten $(\mathrm{W})$ are used in two different tiers) (Batude et al. 2012). Hence, we employ the restriction that M3D-routers to be extended over only two consecutive tiers. As mentioned earlier, Figure 2 shows an illustrative example of M3D-enabled SWNoC architecture, where some routers are extended over at the maximum two tiers. 


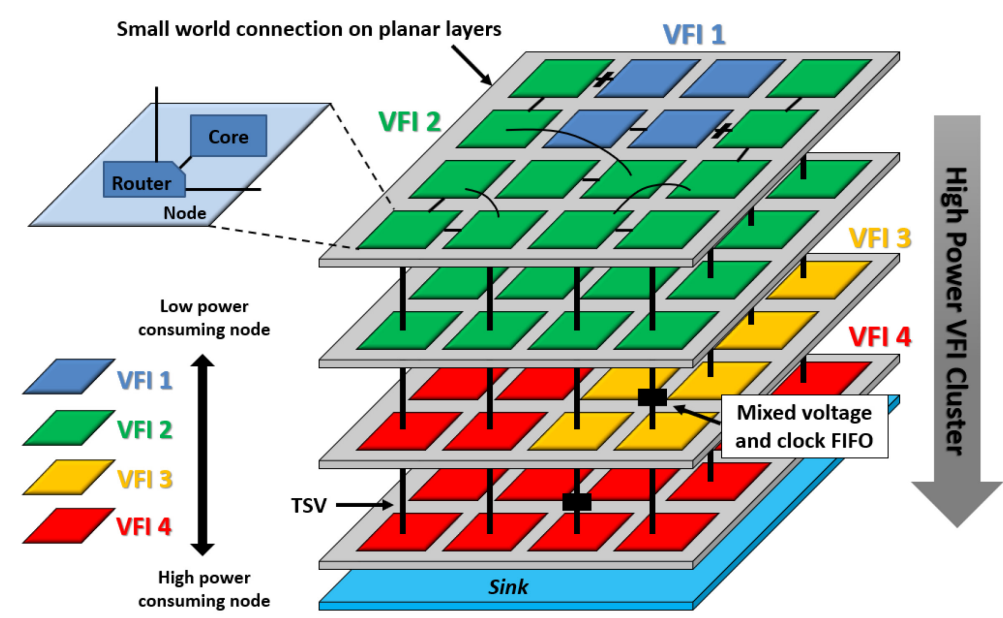

Fig. 3. Illustration of the VFI-enabled 3D manycore chip with small world network-based NoC (SWNoC) architecture. Each VFI has different number of tiles and marked with distinct colors. Mixed voltage and clock FIFOs are placed in the boundary between adjacent VFIs for communication synchronization. TSVs connect tiles from adjacent tiers and enable point-to-point connection. For M3D-enabled SWNoC configuration, the placement of a tile can be extended over multiple tiers and consequently, the placement of the VFI-clusters is completely overhauled compared to the TSV-based design.

\subsection{Voltage Frequency Island-Enabled 3D NoC}

To improve the energy-efficiency of a manycore system without paying substantial performance penalty, VFI-based power management mechanism has become a common practice (Ogras et al. 2009; Jang and Pan 2011). In VFI-based designs, a group of cores and their associated network elements are clustered together based on their computation and communications patterns. Then, each cluster is assigned a single voltage/frequency (V/F) level. The inter-VFI communication between different $\mathrm{V} / \mathrm{F}$ levels is achieved through mixed-clock/mixed voltage first-input first-output (FIFO) interfaces (Chelcea and Nowick 2000). This offers the flexibility to scale the voltage and frequency between various VFIs in order to minimize the overall energy consumption (Ogras et al. 2009). Consequently, in this work, we use VFI as the suitable power management technique in 3D SWNoC and apply heterogeneous clustering (having different number of tiles for each cluster) for exploiting maximum benefits of VFI. The heterogeneous approach has been found to be more efficient than the homogeneous counterpart for enabling VFI-based power management (Kim et al. 2016).

To illustrate the heterogeneous-clustering-based VFI-enabled 3D NoC architecture, Figure 3 shows an example of a 64-core system with four VFIs. As seen in the figure, the number of cores in each individual cluster varies among them. In addition, to ensure the thermal efficiency of the overall chip, the VFI clusters with high-power consuming cores are placed closer to the heat sink for removing maximum possible heat from the system. Consequently, it is possible for thermal efficient architectures that highly communicating cores are separated and placed far from each other, which introduces additional performance penalty.

For a manycore chip, the VFI-based power management can be applied in two ways, viz., Static Voltage Frequency Islanding (SVFI) and Dynamic Voltage Frequency Islanding (DVFI). In this work, our main target is to analyze and quantify how incorporation of VFI-based power management can improve the thermal profiles of the 3D manycore system. Hence, as a case study, we have considered SVFI for the performance-thermal tradeoff analysis undertaken in this work. 
It should be noted that we can also apply DVFI methodology as that improves energy efficiency of the system (Kim et al. 2016). However, as our principal aim is to study the performance-thermal tradeoffs of 3D NoC architectures, we adopt a relatively simpler power management strategy.

To find a suitable clustering, we group the cores that have similar utilization, i.e., instructions per cycle (IPC) and hence can benefit from the same V/F level tuning. For example, cores with low utilization are grouped together and assigned low V/F levels, while cores with high utilization are clustered together to allocate high V/F levels. To determine the best static V/F levels for any cluster, we employ the power and performance models proposed in Juan et al. (2013). The model uses a constrained-posynomial-based formulation for determining the workload-dependent power frequency relationship over an extended range. Also, the performance and power models are trained and cross-validated for various benchmarks with root-mean-squared-percentage-error (RMSPE) of 4.37\% (Juan et al. 2013).

The efficiency of a VFI-based 3D manycore chip significantly depends on the NoC architecture and the associated physical layer design. For a TSV-based 3D chip, tiles from adjacent tiers are only connected by the TSVs in a point-to-point manner. As explained in Figure 2, non-adjacent tiles from adjacent tiers suffers from higher hop count for TSV-based NoCs compared to the M3D counterparts. Consequently, two highly communicating tiles from two different tiers suffer from higher network latency and network energy consumption for TSV-enabled NoCs if these tiles are not placed directly on top of each other. In Figure 3, we have already seen that for a thermally efficient architecture, power-hungry cores (and VFI/s) are placed near the heat sink. Consequently, this placement constraint for TSV-based architectures introduces an additional performance penalty. On the other hand, the M3D-based design does not require this placement constraint due to the absence of the bonding layer and the presence of only an extremely thin ILD layer between adjacent tiers. These physical constraints need to be considered when designing the overall VFI-based architecture.

\section{OPTIMIZATION OF THERMAL-AWARE 3D NOC ARCHITECTURE}

In this section, we present the optimization methodology of our proposed architecture including thermal modeling. First, we address the challenges in designing high-performance 3D NoC with VFI. For exploring the design space of thermal-aware 3D NoC, we introduce the formulation of a new objective function and describe our optimization approach for the target problem. In addition, we also explore the performance and thermal tradeoffs of TSV- and M3D-based NoCs with VFI.

\subsection{Performance and Thermal-Efficient 3D NoC Incorporating VFI}

As mentioned earlier, power density and the resulting thermal issues are the major limitations of TSV-based 3D integration. Incorporating suitable power management strategy improves the power and thermal profiles of the TSV-based 3D NoC. Voltage-Frequency Island (VFI)-based power management is a well-known mechanism to lower the overall energy consumption of a manycore chip within a given performance constraint (Ogras et al. 2009; Jang and Pan 2011). Hence, it has been employed to handle the thermal hotspots of conventional 2D as well as 3D manycore chips (Kumar et al. 2014a).

However, most of the existing VFI methodologies for TSV-based 3D NoC ignores the performance-thermal tradeoffs (Seiculescu et al. 2010; Rahmani et al. 2013). For VFI-based system, a group of cores with similar communication and computation characteristics are clustered together. A group of cores and the associated network elements forming a cluster needs to be physically placed together. Hence, for the thermal-aware 3D design, this placement of the VFI cluster introduces additional constraints for optimizing the thermal profile. Hence, the position of the VFI clusters affects both performance and thermal profiles of the 3D NoC. To design a 
high-performance and thermally efficient 3D NoC with VFI-based power management, we need to consider two aspects. First, the thermal model of the 3D configuration needs to be accurate, and second, a suitable optimization methodology is required to ensure achieving high performance without introducing thermal bottlenecks. We elaborate the design of thermal-aware 3D NoC architecture with VFI power management in the following sections.

\subsection{Thermal Modeling}

As mentioned earlier, high-performance 3D manycore chips suffer from high power density and thermal issues. To overcome such challenges, incorporation of VFI-based power management strategy improves both the power and thermal profiles of the 3D NoC-based manycore chips further. In this work, we also employ VFI-methodology as the suitable power management mechanism in 3D SWNoC and apply heterogeneous clustering (having different number of tiles for each cluster) for exploiting maximum benefits of VFI. In order to improve the thermal profile and achieve maximum benefits of VFI-incorporated architecture, the placement of cores and links plays a significant role. Hence, we optimize the locations of the cores and links at the same time to achieve optimum configuration both from a performance and thermal perspective. To unify the NoC performance with thermal-aware core and link placement, we define a single cost function that combines them as follows:

$$
\cos t=\alpha \cdot O+\beta \cdot T
$$

where $O$ is the unified NoC performance from (1), Tis the temperature of the system, and $\alpha$ and $\beta$ are weighting factors. Our goal is to minimize the cost function to achieve the optimum network latency, energy consumption, and the lowest temperature of the 3D SWNoC. Hence, the optimized architecture ensures high performance and thermal and energy efficiency at the same time.

In order to estimate the temperature T, the 3D IC along with the NoC components are converted into a $3 \mathrm{D}$ resistive network (Cong et al. 2004). Then, we can solve the whole 3D resistive network by a matrix solver to obtain the temperature of each node. Additionally, the standard thermal profile characterization tool, e.g., Hotspot simulator (Huang et al. 2006) can be employed to determine the temperature, T. However, solving the matrix equation (and/or the standard thermal simulator call) for each solution during the optimization process is still costly in terms of resource and time consumption. Hence, we use the established model from Cong et al. (2004), which is simple and relatively fast to make the optimization methodology faster.

This model considers the vertical heat flow and horizontal heat flow separately. When considering only the vertical heat flow, the manycore system can be divided into $n$ single-tile stacks, each with $k$ layers, where $n$ is the number of tiles on a single layer and $k$ is the total number of layers. The temperature of a core within a single-tile stack $n$ located at layer $k$ from the sink $\left(T_{n, k}\right)$ due to the vertical heat flow is given by:

$$
T_{n, k}=\sum_{i=1}^{k}\left(P_{n, i} \sum_{j=1}^{i} R_{j}\right)+R_{b} \sum_{i=1}^{k} P_{n, i},
$$

where $P_{n, i}$ is the power consumption of the core at layer $i$ from the sink in single-tile stack $n, R_{j}$ is the thermal resistance in vertical direction, and $R_{b}$ is the thermal resistance of the base layer on which the dies are placed (Cong et al. 2004). The values of $R_{j}$ and $R_{b}$ are extracted through hotspot simulations (Huang et al. 2006). The value of $R_{j}$ for M3D-based design is lower than the TSV-based counterpart due to the material and structural differences. Hence, the locations of the cores have lower impact on the temperature of the M3D-based design compared to that of TSV.

When considering the horizontal heat flow, high-power tiles should be spread out throughout the planar layer to avoid a hotspot. This is represented through the maximum temperature 
difference at the same layer $k(\Delta T(k))$ (Cong et al. 2004):

$$
\Delta T(k)=\max _{n} T_{n, k}-\min _{n} T_{n, k}
$$

Combining both horizontal and vertical heat-flow models, our objective becomes minimizing the maximum temperature calculated by (3) weighted by the maximum temperature difference (4).

\subsection{Thermal-Aware Optimization}

To ensure thermal- and performance-aware-efficient architecture, our target is to optimize the overall cost function defined in (2) by exploring the link, core, and router placements. While any kind of optimization is suitable for this purpose, however, we use simulated annealing (SA) algorithm for its simplicity and popularity in electronic and VLSI-related design automation (Bui et al. 1989; Chen and Chang 2005). As we focus on both core and link perturbation in the 3D SWNoC architectural space, we utilize two moves for solution perturbation namely the swapping $(S(i, j))$ and the new link placement $(L(l))$ as discussed below:

- Swapping - S(i,j). Selects two cores $i$ and $j$ randomly, and swaps their locations. By swapping the placement of two cores, the communication pattern in the network changes and hence, the NoC configuration is changed as well. Consequently, it results in a different numerical value for the objective function before and after the "swapping" function is invoked. It is to be noted the swapping perturbation function ensures similar network constraints are maintained before and after the function is invoked.

- New Link Placement - L(l). Selects and removes a link of length $l$ between two randomly selected routers. If the link is a planar link, this function creates a new link of the same length between two other routers in the same layer so that overall link distribution remains same.

The $L(l)$ move preserves the number of length $l$ links, which is required to maintain the powerlaw and the small-worldness of the 3D SWNoC architecture during and after the optimization. On each iteration, the probability of selecting the $S(i, j)$ or the $L(l)$ perturbation is set to 0.5 . Alternating in between these two perturbations, we avoid the possibility of getting stuck to a local minimum in the solution space and thereby reaching a (near-) global optimum.

To incorporate the VFI-based power management methodology and to optimize the architecture, we modify the simulated annealing algorithm used for thermal aware optimization as described above. First, we modify the swapping perturbation function, $S(i, j)$ to $S^{\prime}(i, j)$ as follows:

- VFI Swapping - $S^{\prime}(i, j)$ : selects two cores $i$ and $j$ randomly and swaps their locations if the locations do not violate the constraint of VFI clusters after swapping. Consequently, VFI swapping function $S^{\prime}(i, j)$, is a constrained version of the original swapping function $S^{\prime}(i, j)$.

By redefining the swapping move $\left(S^{\prime}(i, j)\right)$, we can optimize the location of cores and VFI clusters with the simulated annealing-based algorithm. In the following example, we show how the VFI swapping function works. For swapping in a VFI-enabled architecture, we select two cores $i$ and $j$ randomly and swap their location if their locations do not violate the constraint of VFI clusters after swapping (no cores are placed isolated from their parent V/F clusters). For example, Figure 4 illustrates an example of a VFI-enabled 3D NoC, which has been marked with the distinct colors to highlight the different VFI clusters and the types of swapping moves for this configuration that are allowed. Figure 4(a) shows the initial placement of the VFI clusters and individual tiles. In Figure 4(a), if we swap two cores, viz. A and B, there is no isolated cores from their respective V/F clusters after swapping A and B as shown in Figure 4(b). Consequently, no violation of the VFI constraint occurred, and hence, the corresponding swapping is allowed. However, if we swap 


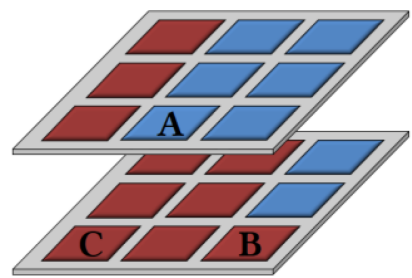

(a)Initial design

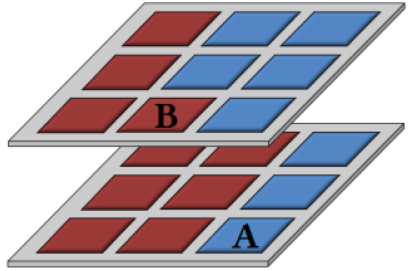

(b)After swapping A and B

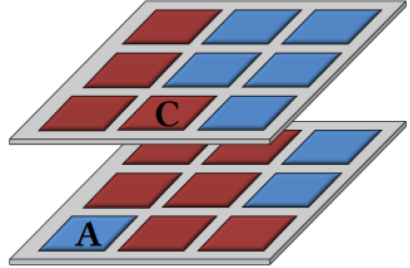

(c)After swapping A and C

Fig. 4. Illustration of the core swapping moves for optimizing the VFI-enabled architecture. (a) The initial VFI-enabled 3D design before any kind of swapping is invoked. (b) After swapping the cores A and B, no placement violation occurred for VFI clusters (swapping is allowed). (c) Swapping the cores A and C introduces placement violation VFI clusters as the core A is isolated from its original VFI-cluster (blue-color cluster) (hence, this swapping is not allowed).

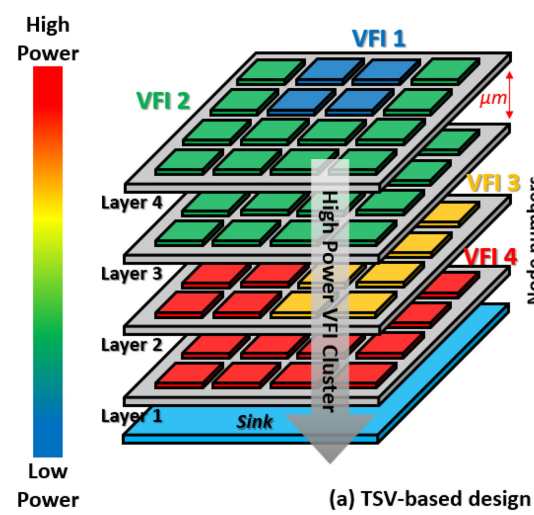

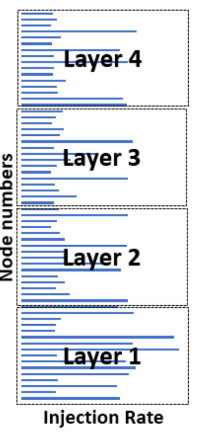

(a) TSV-based design
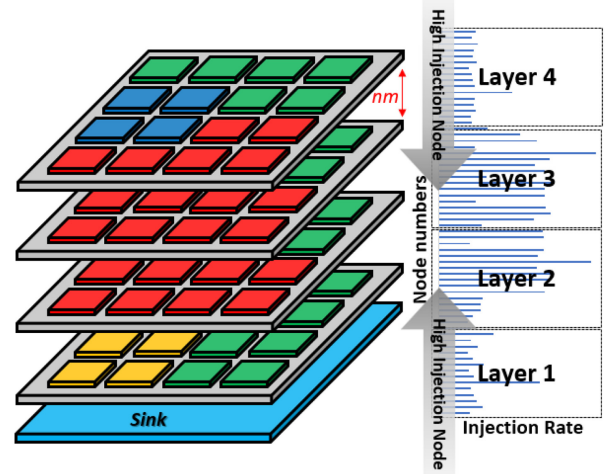

(b) M3D-based design

Fig. 5. Illustration of the relation among the placements of cores, their traffic injection rates, and overall power consumption profile for TSV- and M3D-based 3D NoC architectures with VFI configuration. Different elements are marked with different colors to emphasize the relative power consumption profiles. (a) For a TSV-based design, high-power consuming cores are placed near the heat sink while highly communicating nodes are not exactly located adjacent to each other. (b) For an M3D-based design, highly communicating cores are placed together in the middle two-tiers regardless of their power consumption profile.

cores A and C in Figure 4(a), core A is isolated from blue clusters as shown in Figure 4(c). This results in the violation of the VFI-placement constraint and hence, this swapping is not allowed.

Figure 5 shows the VFI cluster configurations of four-layer 3D NoC architecture using TSV- and M3D-integrations. The injection rates of each node, shown along the vertical bar, refer to the internode communication patterns. As shown in Figure 5(a), during the optimization process for TSVbased design, optimizing the cost function (as defined in (2)) places the high-power consuming cores (cores and network elements in the red-color VFI cluster) close to the bottommost layer (near the heat sink) while low-power consuming cores (blue-color VFI) move to the topmost layer. Therefore, the high-power VFI cluster is placed closer to the heat sink to improve the thermal profiles. Hence, even if we put the high-power VFI cluster away from the heat sink in the initial design, for the optimized configuration, the cluster will move near the heat sink regardless of the initial VFI-cluster placement. In addition, it is also seen that for this TSV-based optimized architecture, some of the highly communicating cores are not placed near each other as seen from 
their core placement and respective traffic injections rates. On the other hand, for M3D-based design, the temperature term $(\beta \cdot T)$ in Equation (2) is not strongly affected by the locations of the VFI clusters due to low thermal resistance $\left(R_{j}\right)$ and the thin ILD layer. Hence, the thermal constraint is relaxed when optimizing the M3D-based architecture with VFI. Consequently, for the optimized architecture shown in Figure 5(b), we can see that the VFI clusters with high power are not placed adjacent to the heat sink. However, for inter-VFI communication, the clusters with highly active and frequently communicating cores are placed in the middle layers. As the highly communicating nodes are placed in the adjacent middle layers, they communicate among themselves with reduced hop counts (in most of the cases one or two hops) and thereby improving the performance as these configurations need not follow the strict placement constraints for thermal efficiency like the TSV-based counterparts. It is to be noted that the number and placement of the multi-tier routers affect the overall NoC performance. Depending on the application characteristics, the placement of VFI clusters and multi-tier routers varies. The optimization methodology determines the (near-) optimal placement of both the multi-tier routers and VFI-cluster simultaneously by considering their effects on the NoC performance and the thermal profile.

\section{EXPERIMENTAL RESULTS AND ANALYSIS}

In this section, we compare the performance and temperature profiles of the M3D- and TSVenabled SWNoC architectures. We first describe the experimental setup adopted in this work. Second, we present a comparative performance evaluation between TSV- and M3D-based integrations by varying the number of layers. Next, we analyze the performance-thermal tradeoffs between the joint optimized (performance and thermal) and the performance-oriented TSV-based 3D SWNoCs with VFI configuration. Finally, we evaluate the effectiveness of the joint optimized TSV- and M3D-based NoC designs by incorporating VFI techniques.

We consider two relevant performance metrics: latency, and energy-delay-product (EDP). We define the EDP as the product of network latency and energy dissipation to unify the effects of both these two metrics into a single parameter. To evaluate the thermal profiles of the architecture, we consider the maximum, average, and minimum temperatures of the manycore chip.

\subsection{Experimental Setup}

To obtain the detailed processor and network level information, we use GEM5 (Binkert et al. $2011)$, the full system simulator, for a system consisting of $64 \times 86$ cores. The memory system is MOESI_CMP_directory and it consists of 64KB L1 (data + instruction) and a shared 8MB L2 cache. Due to the irregular nature of the 3D SWNoC architecture, the topology-agnostic Adaptive Layered Shortest Path Routing (ALASH) is used for data exchange (Lysne et al. 2006). In this performance evaluation, we consider a set of eight applications with widely varying communication and computation patterns. We use four SPLASH-2 benchmarks (FFT, RADIX, LU, and WATER) (Woo et al. 1995) and four PARSEC benchmarks (DEDUP, VIPS, FLUIDANIMATE (FLUID), and CANNEAL (CAN)) (Bienia 2011). We incorporate GEM5 statistics in McPAT (Multicore Power, Area, and Timing) (Li et al. 2009) to determine the power consumption.

We follow the hybrid clustering methodology presented in Kim et al. (2016). Table 1 shows the $\mathrm{V} / \mathrm{F}$ values of all four VFI clusters and their respective sizes for the eight benchmarks. These VFI clusters are created to achieve at least $95 \%$ performance of the system running with the nominal V/F. We use the hotspot (Huang et al. 2006) to obtain the thermal profile of the whole system. We arrange the 64 cores in one-layer, two-layer, three-layer, and four-layer 3D systems. Table 2 shows the die size of each different layer structures. In this work, we place the cores and routers in a regular grid pattern. Therefore, in two- and four-layer configuration, the cores, and routers are placed in $4 \times 8 \times 2(=64)$ and $4 \times 4 \times 4(=64)$ grids. In the three-layer configuration, the cores and 
Table 1. VFI Clusters Size and Their Respective V/F levels (Kim et al. 2016)

\begin{tabular}{|c|c|c|c|c|c|c|c|c|}
\hline & CAN & DEDUP & FFT & FLUID & LU & RADIX & VIPS & WATER \\
\hline Cluster 1 & $22-0.6$ & $40-0.9$ & $29-0.9$ & $40-0.9$ & $32-0.8$ & $37-1.0$ & $30-0.7$ & $33-0.8$ \\
\hline Cluster 2 & $22-1.0$ & $16-1.0$ & $23-1.0$ & $16-1.0$ & $24-1.0$ & $19-0.9$ & $26-0.9$ & $23-1.0$ \\
\hline Cluster 3 & $16-0.6$ & $4-1.0$ & $7-0.9$ & $4-0.7$ & $4-0.6$ & $4-0.9$ & $4-0.7$ & $4-0.9$ \\
\hline Cluster 4 & $4-0.9$ & $4-1.0$ & $5-0.9$ & $4-0.8$ & $4-0.9$ & $4-0.8$ & $4-0.9$ & $4-0.7$ \\
\hline
\end{tabular}

Table 2. Chip Size for the Different Number of Layers

\begin{tabular}{|l|c|c|c|c|}
\hline Number of layers & 1 & 2 & 3 & 4 \\
\hline Layer size $\left(\mathrm{mm}^{2}\right)$ & $20 \times 20$ & $20 \times 10$ & $15 \times 10$ & $10 \times 10$ \\
\hline
\end{tabular}

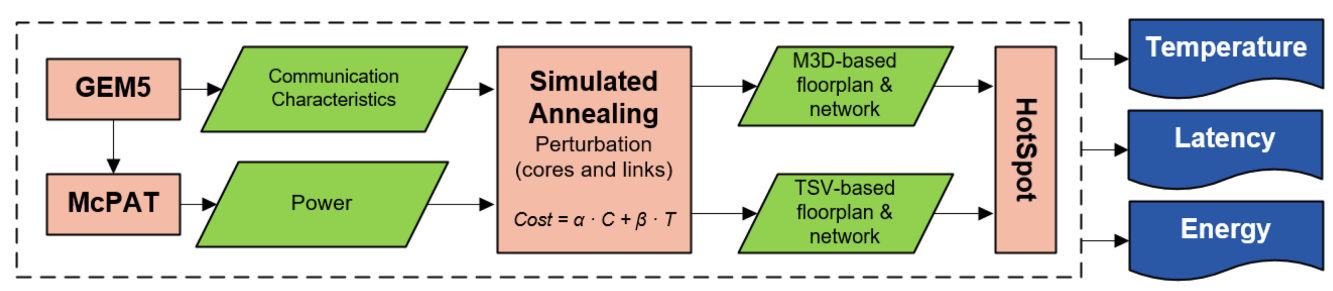

Fig. 6. Experimental set up for establishing the performance and thermal tradeoff for TSV- and M3D-based NoC architectures.

routers are placed in a $4 \times 6 \times 3(=72)$ grid that incorporates more than 64 cores. Hence, we use eight dummy blocks for the rest of area. They are placed on the edge of each die so that they do not affect the communication distance between nodes and the overall optimization methodology. All the SWNoC configurations use the same number of vertical and planar links as a 3D MESH. Figure 6 shows our overall experimental setup.

\subsection{Comparative Performance Evaluation of TSV- and M3D-Based NoCs}

In this section, we compare the performance and thermal profiles of the TSV- and M3D-enabled SWNoC architectures (optimized only for performance). In particular, we evaluate the impacts of the TSV- and M3D-based integrations and the number of planar layers.

Figure 7 demonstrates the normalized network latency of the TSV- and M3D-based SWNoCs without incorporating any power management for varying number of layers. All the network latencies are normalized with respect to that of 2D SWNoC (one-layer configuration). In this figure, it is evident that as the number of layers increases, the network latencies of TSV- and M3D-based SWNoCs decrease. The best network latency value is achieved for the four-layer configuration. To explain this, Figure 8 shows the normalized average path lengths (as defined in (1)) for TSVand M3D-based designs. As seen in this figure, the M3D-based SWNoC achieves lower communication path length than the TSV-based configurations for the same number of layers. Hence, M3D achieves lower network latency than the TSV-based counterpart. In addition, increasing the number of planar layers lowers the average path length significantly. For example, a four-layer 3D SWNoC architecture can reduce as much as $15.0 \%$ and $31.1 \%$ average path length for TSVand M3D-based SWNoC architectures respectively compared to their one-layer counterparts. As 


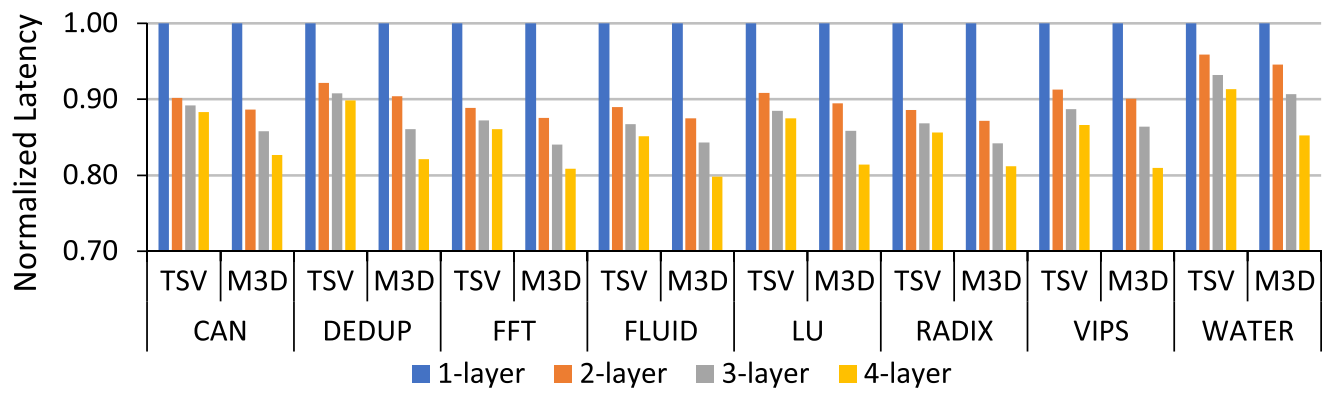

Fig. 7. Normalized network latency of the TSV- and M3D-based SWNoCs with varying number of planar layers without incorporating any VFI-based power management.

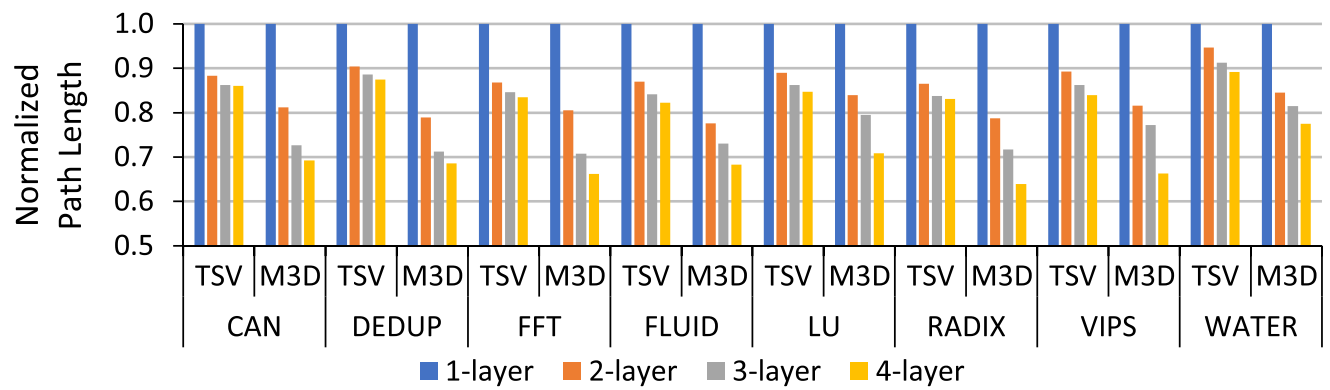

Fig. 8. Normalized path length of the TSV- and M3D-based SWNoCs with varying number of planar layers without incorporating any VFI-based power management.

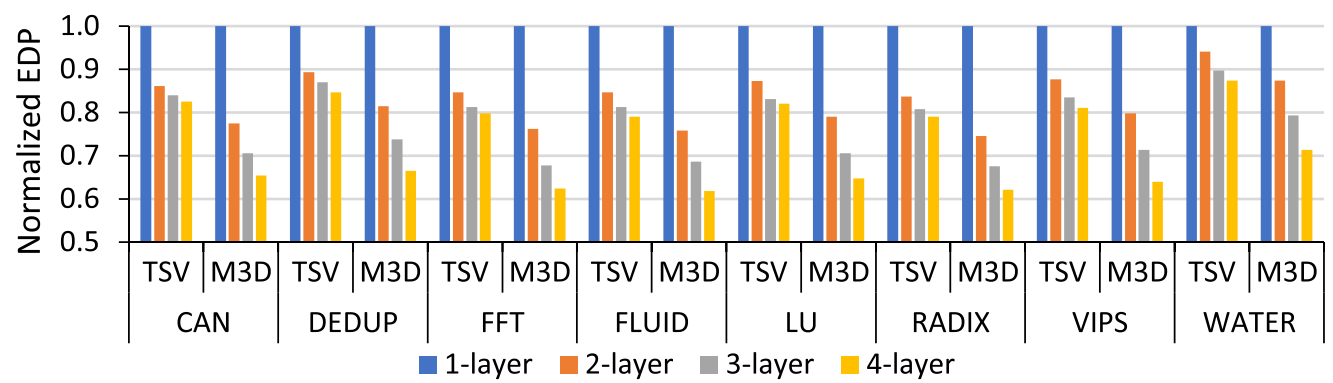

Fig. 9. Normalized EDP profiles of the TSV- and M3D-based SWNoCs with varying number of planar layers without incorporating any VFI-based power management.

a result, the four-layer TSV- and M3D NoC architecture shows $12.4 \%$ and $18.2 \%$ lower network latencies compared to their one-layer counterpart.

Figure 9 shows the EDP profile of the TSV- and M3D-based SWNoCs for different number of planar layers. In this figure, we observe that as the number of layer increases, the EDP value decreases for both the TSV- and M3D-based architectures. The EDP value of a network depends on the average hop count and communication path length. As seen in Figure 8, with increasing number of layers in the chip, more numbers of vertical links can act as short cuts between long-distant nodes from different layers and the path length reduces. Thus, following the network latency trend in Figure 7, the EDP also decreases progressively as the number of planar layer increases. The 3D NoC with additional layers offers more connectivity and larger design space for 


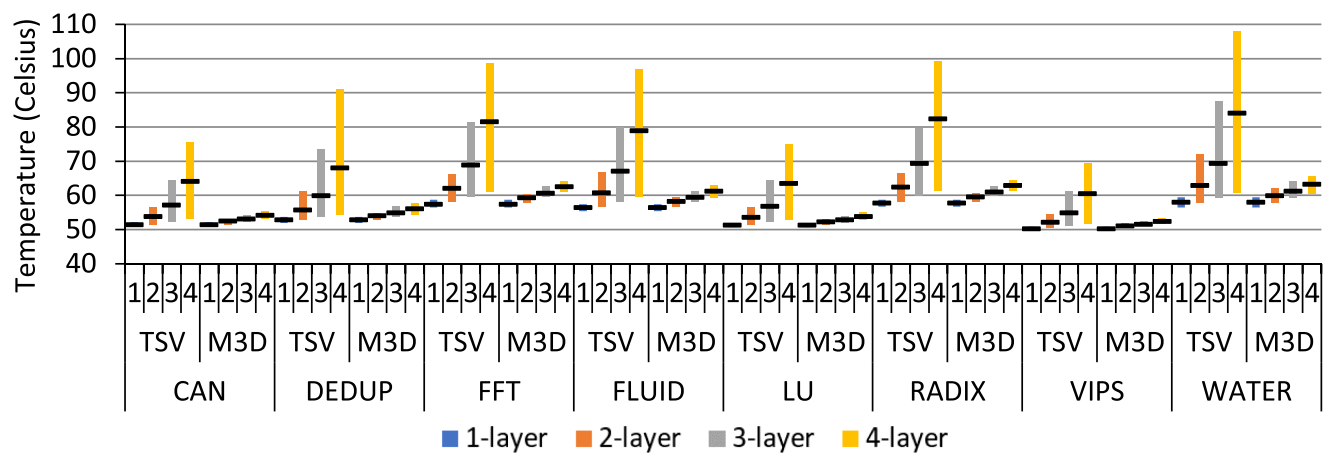

Fig. 10. Maximum, average and minimum temperature values of the TSV- and M3D-based SWNoCs with varying number of planar layers without VFI power management.

optimization. Therefore, 3D NoCs with a higher number of layers achieve better EDP profiles than 2D NoCs (number of layer equals to 1 for 2D NoC). The TSV-based 3D SWNoC with four-layer shows up to $20.9 \%$ (average 18.1\%) lower EDP than 2D SWNoC while the M3D-based SWNoC with four-layer shows up to $31.6 \%$ (average 28.7\%) lower EDP than the 2D configuration.

In addition, in Figure 9, by comparing the EDP of TSV- and M3D-based architectures for all possible configuration, we can see that the M3D outperforms TSV-based counterparts while using the same number of layers. Compared to the TSV-based 3D SWNoC, the M3D achieves an average of $0 \%, 8.2 \%, 12.6 \%$, and $17.2 \%$ reduction in EDP for one-layer, two-layer, three-layer, and fourlayer implementations, respectively. This happens because the high-energy consuming TSVs are replaced by energy-efficient MIVs. While the energy consumption of MIVs is comparable to that of planar metal wires, the associated energy per bit for the TSVs are higher. Depending on the implementation methodology and technology parameters, the values of the typical TSV energy per bit are 4 5 times more than that of metal wires (Lee et al. 2013; Samal et al. 2016). Also, for M3D-enabled NoC, the routers are extended over multiple tiers and consequently, the values for both the average hop count and path length are reduced compared to the TSV-based design.

Figure 10 shows the maximum, average, and minimum temperatures of the TSV- and M3D-based SWNoCs with a varying number of planar layers without any power management. In this figure, we observe that the temperature increases for TSV- and M3D-enabled SWNoC architectures as the number of layers increases. Although the power consumption of the $3 \mathrm{D}$ NoC is expected to decrease due to the considerably shorter connections, the power density will increase since there is a larger number of devices per unit area as compared to planar 2D NoC as the number of layer increases. Hence, the increased power density results in higher temperature profiles. To evaluate the impact of different 3D fabrication technologies, we compare the temperatures between the TSV- and M3D-enabled SWNoC. The M3D NoC architecture improves the temperature profiles compared to the TSV-based SWNoC.

The maximum temperature of an M3D-based four-layer SWNoC architecture is reduced by up to $39.1 \%$ (average $32.2 \%$ ) compared to the TSV-based counterpart. In addition, the difference between maximum and minimum temperatures of TSV-based NoC architectures of one-layer, two-layer, three-layer and four-layer configurations are $1.8^{\circ} \mathrm{C}, 7.9^{\circ} \mathrm{C}, 18.2^{\circ} \mathrm{C}$, and $32.3^{\circ} \mathrm{C}$, respectively. On the other hand, the difference between maximum and minimum temperatures of M3D-enabled NoC architectures of one-layer, two-layer, three-layer, and four-layer configurations are $1.8^{\circ} \mathrm{C}$, $2.3^{\circ} \mathrm{C}, 2.7^{\circ} \mathrm{C}$, and $3.0^{\circ} \mathrm{C}$, respectively. The temperature difference of TSV-based NoC architectures increases progressively from one- to four-layers and shows much greater difference than the M3D. 


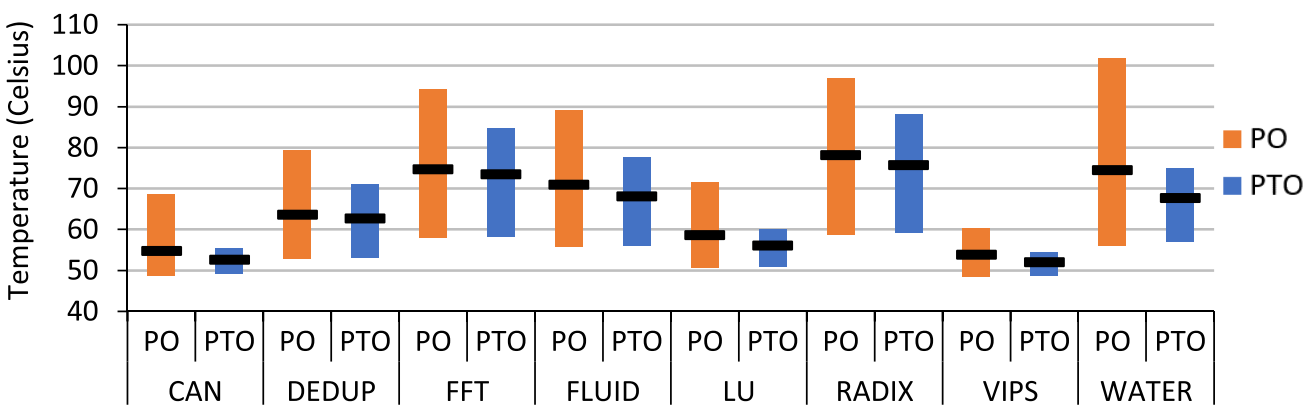

Fig. 11. Maximum, average and minimum temperature profiles of the four-layer PO- and PTO-based TSVenabled 3D NoCs incorporating VFI-based power management for all the benchmarks.

As the number of layer increases, the rate of temperature increase in M3D is much lower than the TSV-based design. This happens because, compared to the TSV-based design, the M3D does not have a bonding layer that has poor thermal conductivity. Also, the extremely thin ILD present between the tiers in M3D-based integration helps smooth thermal flow between tiers. Hence, the M3D-enabled configuration is less prone to the formation of any thermal hotspot.

In addition, for characterizing the thermal profile of manycore chip, thermal throttling has been adopted in previous works (Rao and Vrudhula 2007; Kumar et al. 2014b). In this work, we set the limit on the chip temperature to be $110^{\circ} \mathrm{C}$ (Rao and Vrudhula 2007). As seen in Figure 10, the maximum temperatures of the chip in both TSV- and M3D-based designs are well within the maximum allowable temperature and for most cases, it is under $100^{\circ} \mathrm{C}$. For the WATER benchmark, the maximum temperature rises to $107^{\circ} \mathrm{C}$, which still remains below the allowable limit. Hence, thermal throttling is not required for the applications considered in this work. However, thermal throttling will be necessary for applications where the chip temperature increases beyond the allowable threshold.

\subsection{Evaluation of VFI-Enabled TSV-Based 3D NoC}

In this section, we analyze the performance-thermal tradeoffs between the joint optimized (performance and thermal) and the performance-only optimized TSV-enabled 3D SWNoC. To characterize both the performance and the thermal profile, we consider three parameters, viz. the network latency, energy-delay-product (EDP) per message and the temperature of the system. Also, we consider the maximum, average, and minimum temperatures in order to determine possibility of creating thermal hotspots. For ease of referencing, the joint optimized (performance and thermal) SWNoC and the baseline SWNoC (optimized only for performance) are marked as PTO and PO, respectively. It is to be noted that for the PO-based SWNoC architecture, we optimize the cost function termed as the communication path length, as defined in (1). In contrast, for PTO-based architectures, we target to optimize the joint cost function that unifies both the performance and the temperature of the $\mathrm{NoC}$ as defined in (2).

Figure 11 shows the maximum, average, and minimum temperatures of the four-layer POand PTO-based TSV-enabled 3D SWNoC incorporating VFI-based power management for the SPLASH-2 and PARSEC benchmarks under consideration. In the figure, we can see that the temperature values of PTO-based design are lower than the PO-based counterpart. PTO-based design improves the temperature values compared to $\mathrm{PO}$ across all the benchmarks. The maximum temperature of the PTO-based design is reduced on average 16.9\% compared to the PO-based design. 


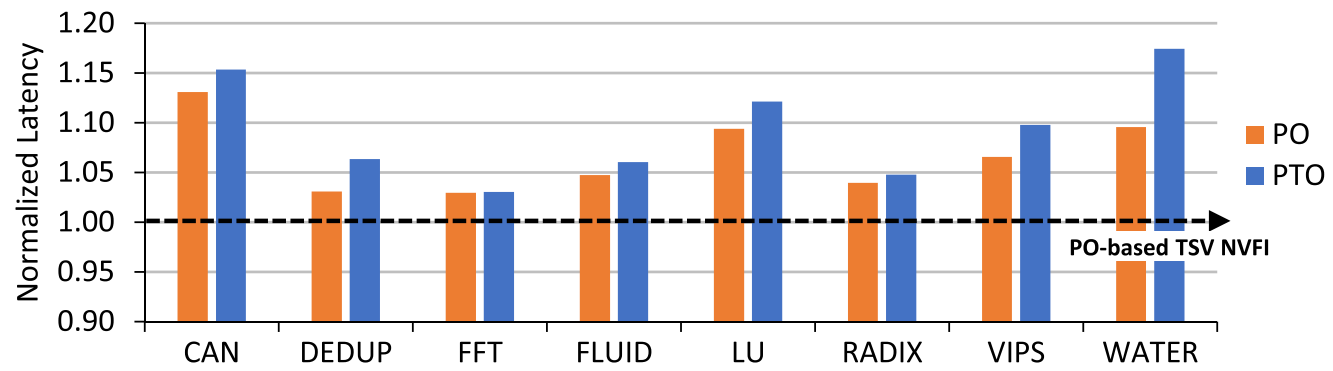

Fig. 12. Normalized network latency of the four-layer PO- and PTO-based TSV-enabled 3D NoCs incorporating VFI-based power management for all the benchmarks.

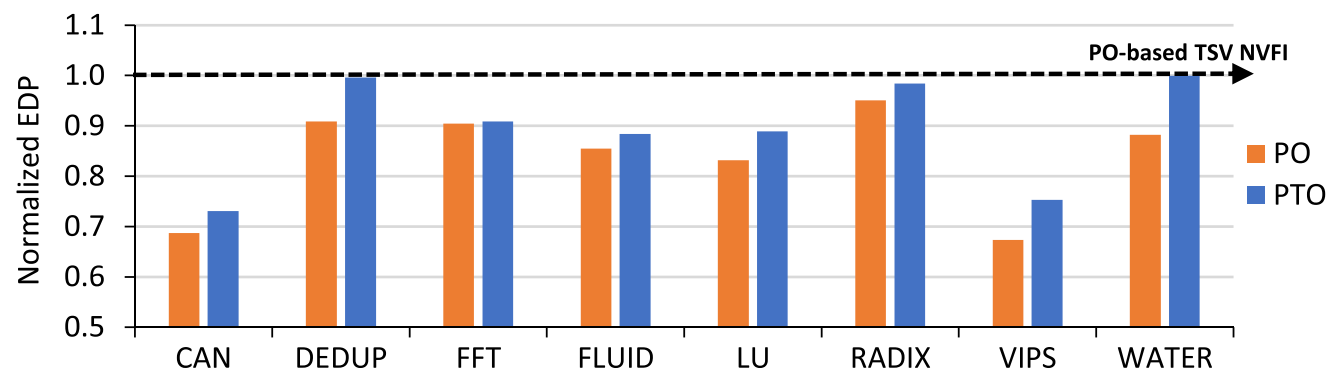

Fig. 13. Normalized EDP of the four-layer PO- and PTO-based TSV-enabled 3D NoCs incorporating VFIbased power management for all the benchmarks.

In addition, the difference between the maximum and minimum temperatures of the PTO-based design is lower than that of the PO-based design.

To consider the tradeoff between the performance and temperature, we also show the network latency and EDP profiles of four-layer PO- and PTO-based TSV-enabled 3D SWNoC in Figures 12 and 13, respectively. The network latency and EDP are normalized with respect to that of PO-based SWNoC without any VFI (NVFI). As seen in Figure 12, the VFI-enabled NoC has higher network latency compared to the NVFI. The network latency of the PO-based TSV-enabled 3D SWNoC incorporating VFI is higher than that of the PTO-based counterpart by up to $7.8 \%$ depending on the specific benchmark. As seen in Figure 13, the PTO-based solution shows up to $11.7 \%$ higher EDP values compared to the PO-based configuration. Moreover, the EDP of DEDUP and WATER benchmarks are degraded almost the same as PO-based TSV-enabled 3D SWNoC without VFI. The variations in network latency and EDP shown in Figures 12 and 13 are related to the injection rates of each node for a particular application.

To explain this, we show the internode traffic intensities for PO- and PTO-based 64-core system for WATER and FFT benchmarks in Figures 14(a) and 14(b), respectively. As seen in Figures 12 and 13, the WATER benchmark has the highest latency and EDP degradation. This happens due to the placement of a few highly active and frequently communicating cores. In Figure 14(a), we see that for the PO-based system, the highly active cores occupy the middle layers where most of the links are concentrated. To optimize the thermal profile, all the highly active cores are pushed towards the sink in the PTO-based system. Hence, the PTO-based configuration incurs noticeable performance penalty. On the other hand, in Figure 14(b), we can see that the FFT benchmark achieves the least change in network latency and EDP. This happens due to the fact that, for the FFT benchmark, the 


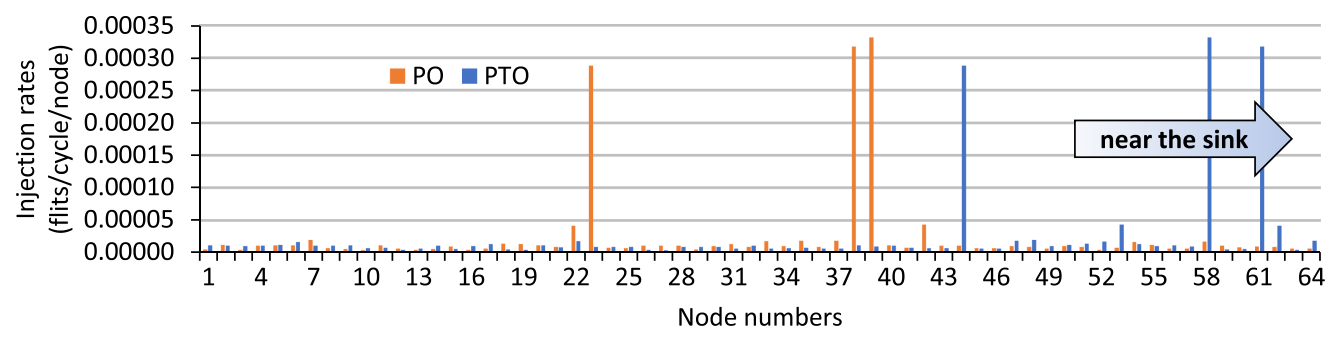

Fig. 14. (a) Internode traffic of each node for PO- and PTO-based 64-core systems with WATER benchmark.

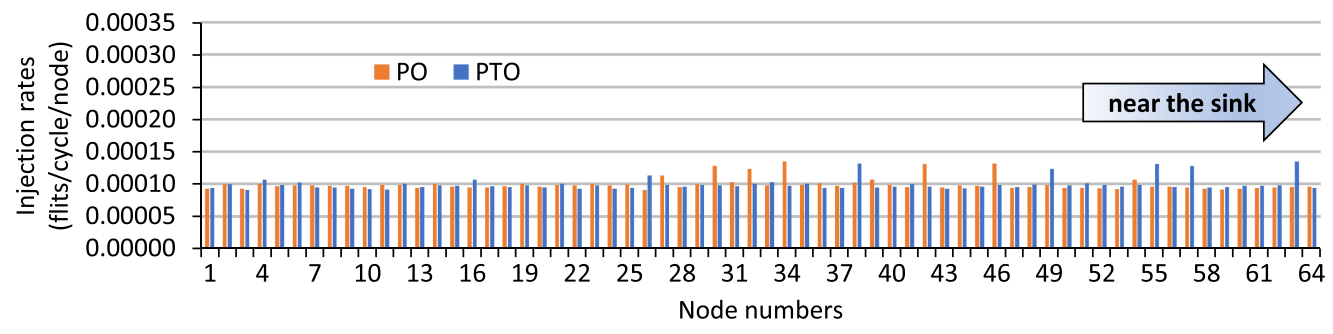

Fig. 14. (b) Internode traffic of each node for PO- and PTO-based 64-core systems with FFT benchmark.

traffic intensities for each node are nearly uniform and thus the latency and EDP of FFT do not degrade significantly by varying the placement of the cores.

By comparing both Figure 12 and Figure 13, one can observe that the PTO technique improves the thermal profile; however, both the network latency and EDP degrade depending on the specific benchmark. The reason behind the increase in latency and EDP of PTO-based design is that a few highly communicating cores with different V/F levels are placed far apart from each other due to the constraint of placing high VFI clusters close to the sink. Hence, PTO-based thermal optimization approach introduces additional constraints on the core placement and consequently, the performance degrades (EDP increases) from PO-based designs.

\subsection{Evaluation of VFI Configuration for TSV- and M3D-Based NoCs}

As described in Section 4.2, we alter the unified cost function and corresponding weight factors which are more suitable for the joint optimized (performance and thermal) of M3D NoCs (referred to as PTM). In this section, we evaluate the performance and thermal profiles of the PTO TSVand PTM M3D-based SWNoC architectures with four-layer design. As inthe previous sections, we choose the normalized network latency, EDP, and the temperature as the relevant metrics.

Figure 15 shows the maximum, average and minimum temperatures of the four-layer PTO TSV- and PTM M3D-based 3D SWNoC architectures. In this figure, the peak temperature of M3D SWNoC architecture reduces up to $29.1 \%$ (average $20.1 \%$ ) compared to the TSV-based configuration. Moreover, the average difference between the maximum and minimum temperatures over all the benchmarks for TSV and M3D designs are $16.7^{\circ} \mathrm{C}$ and $2.9^{\circ} \mathrm{C}$, respectively.

In addition to the thermal benefit, we also evaluate the performance of the PTO TSV- and PTM M3D-based 3D SWNoC architectures. For comparative performance evaluation, the network latency and EDP are normalized with respect to the PTO TSV-based 3D SWNoC architecture. Figure 16 shows the network latencies of PTO TSV- and PTM M3D-based 3D SWNoC. The M3D-based SWNoC reduces the network latency up to $12.9 \%$ (average $8.8 \%$ ) compared to TSV-based design. To illustrate this, Figure 17 shows the normalized average path lengths for each 3D configuration. 


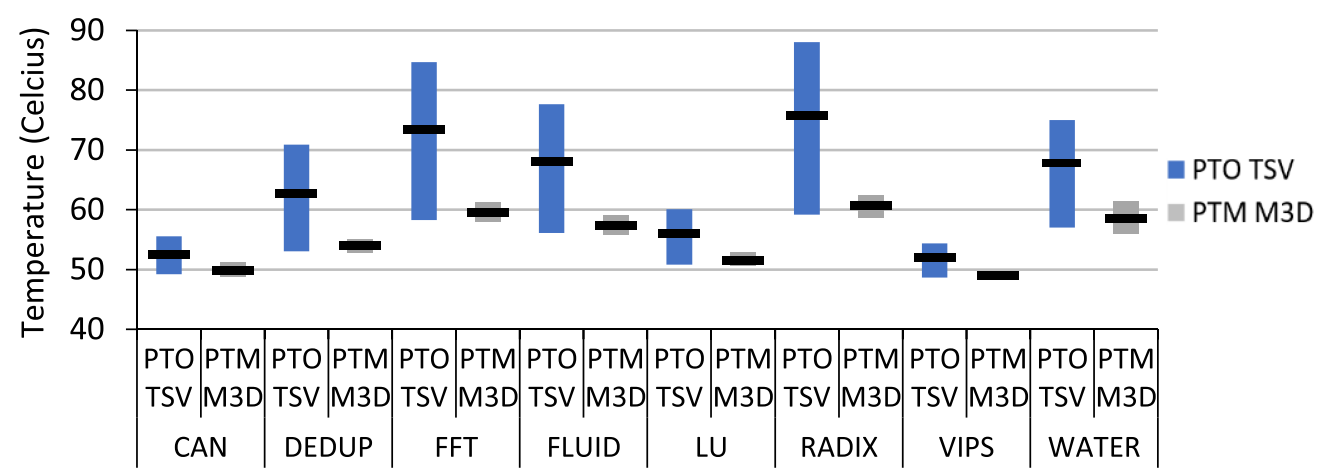

Fig. 15. Maximum, average and minimum temperature values of the PTO TSV- and PTM M3D-based SWNoC architectures with four-layer design.

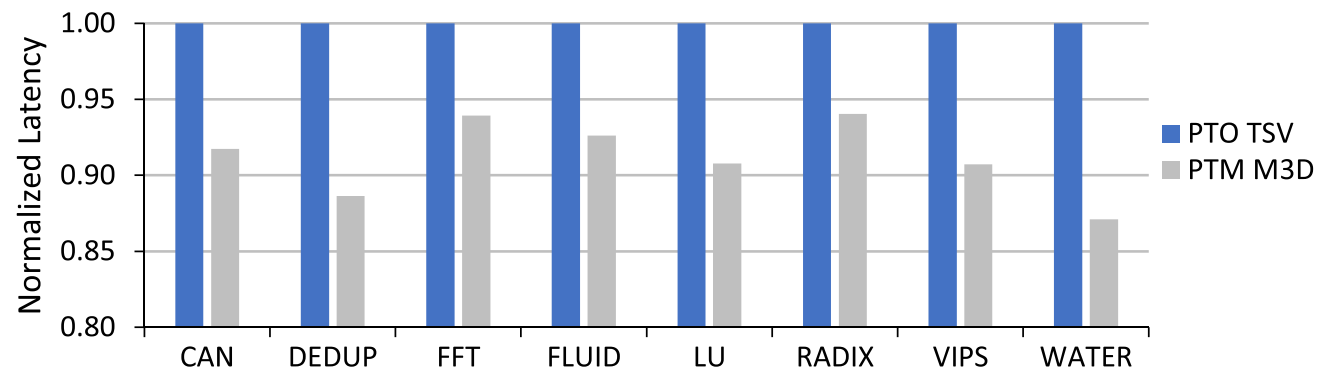

Fig. 16. Normalized network latency of the PTO TSV- and PTM M3D-based SWNoC architectures with four-layer design.

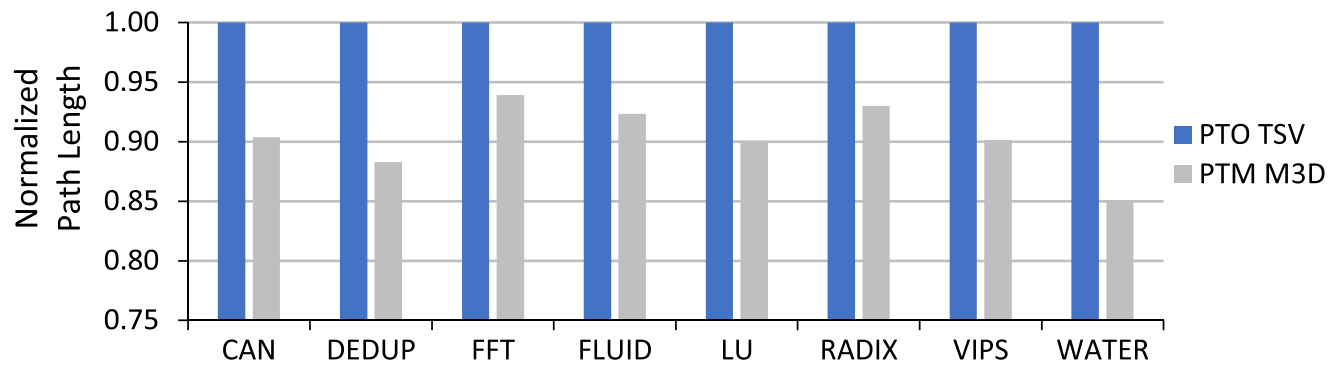

Fig. 17. Normalized path length of the PTO TSV- and PTM M3D-based SWNoC architectures with four-layer design.

On an average, PTM M3D-based SWNoC configuration achieves 9.6\% lower path length compared to the PTO TSV design. Extending a router over multiple tiers in M3D places different ports on different tiers. Hence, router ports belonging to different routers, but placed in the same tiers, can communicate directly without any need to traverse from one planar layer to another, unlike in TSV-based designs. This reduces the path length of an M3D-SWNoC compared to its TSV-based counterpart where all the routers are in a specific planar layer.

We also evaluate the TSV- and M3D-based SWNoCs from the EDP perspective. In Figure 18, the M3D-based design shows up to 29.4\% (average 25.7\%) lower EDP than TSV. The reason behind the decrease in EDP profile of M3D is that the intra-router wire length and the energy consumption 


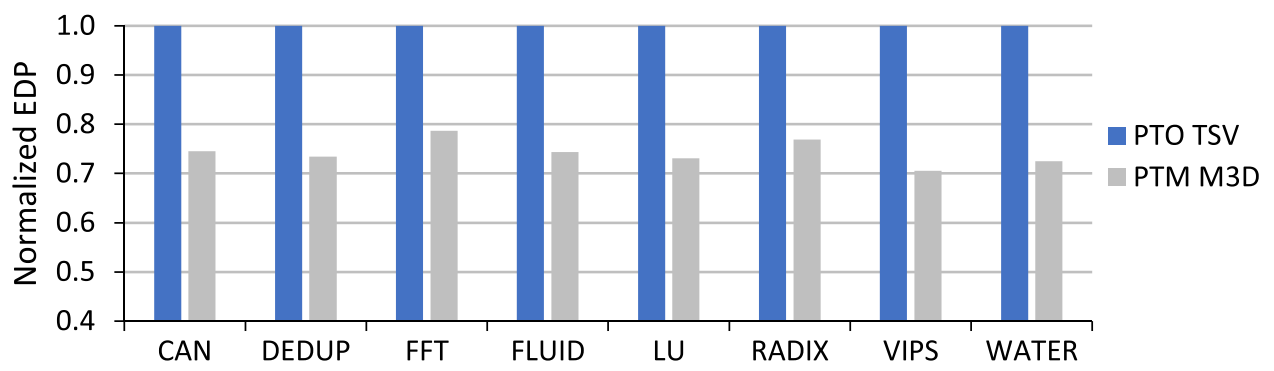

Fig. 18. Normalized EDP profiles of the PTO TSV- and PTM M3D-based SWNoC architectures with 4-layer design.

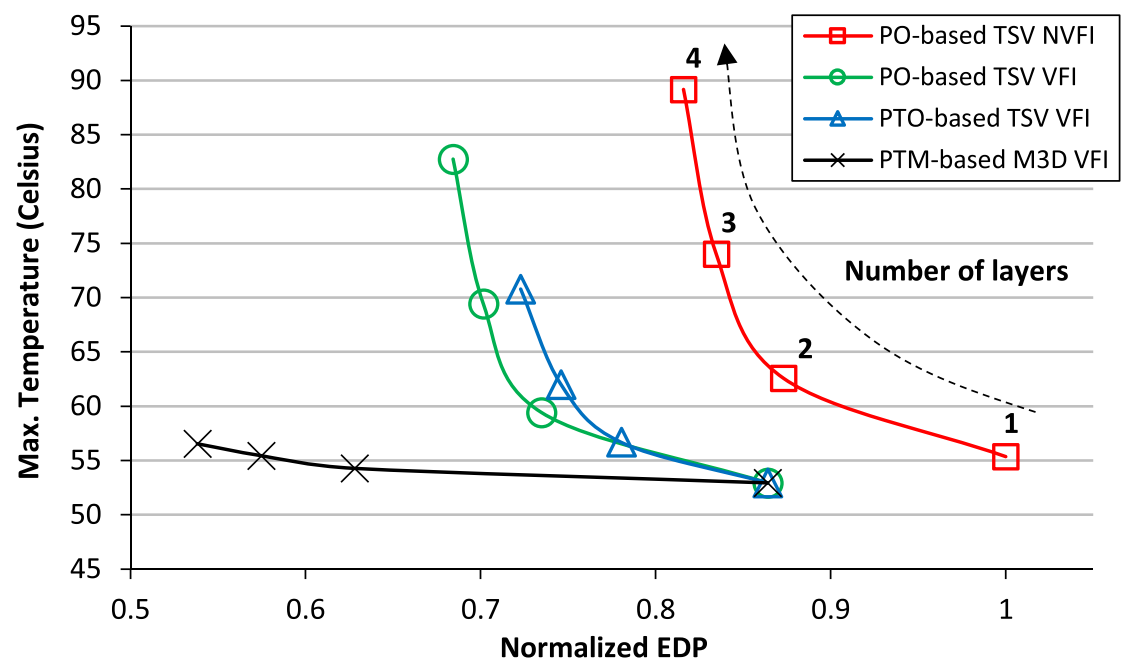

Fig. 19. Average EDP and maximum temperature profiles of PO-based TSV NVFI, PO-based TSV VFI, PTObased TSV VFI and PTM-based M3D VFI (averaged over all benchmarks) for different number of layers. While the maximum temperature increases rapidly for TSV-based configurations as the number of layers increases from one to four, however, for M3D-enabled NoC, the increase of maximum temperature is limited to few $\left(\sim 10^{\circ} \mathrm{C}\right)$ degrees only.

are reduced. In addition to this, TSVs that consume high energy are replaced with energy-efficient MIVs. Finally, we demonstrate that the PTM M3D-based SWNoC shows $29.1 \%$ lower peak temperature than the PTO TSV-based 3D SWNoC. In addition, the M3D-based SWNoC performs $29.4 \%$ better in terms of EDP compared to the PTO TSV-based 3D NoC architecture. The M3D benefits for both thermal and performance profiles without any thermal constraint. Therefore, the M3D can receive the full benefits of power management. We can conclude that PTM M3D-based design is more efficient considering the performance-thermal tradeoffs compared to the PTO TSV-based.

\subsection{Performance and Thermal Tradeoffs for 3D NoC}

To summarize the performance and temperature profiles of all 3D NoC architectures considered here, in Figure 19, we show the variation of average EDP and maximum temperature profiles by changing the number of planar layers. The EDP values are normalized with respect to the EDP value of PO-based TSV 3D NoC without VFI configuration. The maximum temperature is measured 
in degree Celsius as before and plotted along the vertical axis. Four data points on each graph refer to the corresponding one-, two-, three-, and four-layer architectures. Four different configurations that have already been characterized in earlier sections are chosen for summarizing the overall findings of this study. These configurations are PO-based TSV NVFI, PO-based TSV VFI, PTObased TSV VFI and PTM-based M3D VFI configurations.

In this figure, it is evident that the TSV-based VFI-enabled 3D NoC (PO-based TSV VFI) achieves both better performance (lower EDP value) and thermal profiles (lower maximum temperature) for any number of layers compared to their non-VFI counterpart (PO-based TSV NVFI). However, the resultant 3D NoC suffers from possible formation of thermal hotspots as the average of the maximum temperature (averaged over all the benchmarks) rises up to $83^{\circ} \mathrm{C}$. We employ jointoptimization framework (performance and thermal) for 3D NoC architecture (PTO-based TSV VFI). It mitigates the thermal hotspot problem by limiting the average maximum temperature to $70^{\circ} \mathrm{C}$, however, it incurs latency penalty while increasing the average energy consumption by $3 \%$ compared to the PO-based TSV VFI configurations. In TSV-enabled 3D NoCs, stacking more layers improves the achievable performance and energy efficiency of the manycore platforms. However, due to the presence of bonding layer, which is a poor thermal conductor, the maximum temperature of TSV-enabled 3D NoC architectures increases significantly as the number of layer increases from one to four. In contrary, by increasing the number of tiers, the M3D-based design does not increase the maximum temperature as rapidly as TSV-based counterpart. From Figure 19, we can see that the M3D configuration (PTM-based M3D VFI) improves the EDP profile with increasing the number of tiers while the maximum temperature is still much lower than the TSV-based design. For example, for a system configuration of four-layer, PTM-based M3D VFI shows, on average, $17 \%$ lower EDP and $14^{\circ} \mathrm{C}$ reduction in the maximum temperature compared to PTO-based TSV VFI. Therefore, we can conclude that the PTM-based M3D NoC with power management achieves the best performance-thermal tradeoff for the 3D manycore chip.

\section{CONCLUSION}

3D NoCs improve the achievable performance and energy efficiency of the manycore platforms. However, stacking a high number of planar layers while keeping the total foot-print area constant, increases the power density of the system and subsequently aggravates formation of thermal hotspots in the manycore chip. While incorporating the VFI-based power management improves the thermal profile of 3D chip, however, even with power management, in the TSV-based 3D NoCs, the performance improvement is limited due to the thermal constraint. To address these challenges, in this article, we presented the design of M3D-based NoCs incorporating VFI-based power management. The full benefit of M3D integration was explored considering the aforementioned thermal constraints. For M3D-enabled architectures, the routers are extended over multiple tiers using minuscule-sized MIVs and the presence of thin ILD structure between the tiers to provide a high-performance and thermally viable 3D manycore chip. We demonstrate that the M3Dbased SWNoC architecture incorporating VFI-enabled power management achieves up to $29.4 \%$ lower energy-delay-product (EDP) compared to the TSV-based counterparts. Also, the M3D-based NoC shows up to $29.1 \%$ lower maximum temperature than TSV-based design.

\section{REFERENCES}

P. Batude, T. Ernst, J. Arcamone, G. Arndt, P. Coudrain, and P. E. Gaillardon. 2012. 3-D sequential integration: A key enabling technology for heterogeneous co-integration of new function with CMOS. IEEE 7. Emer. Select. Top. Circ. Syst. $2,4,714-722$.

P. Batude, B. Sklenard, C. Fenouillet-Beranger, B. Previtali, C. Tabone, O. Rozeau, O. Billoint, O. Turkyilmaz, H. Sarhan, S. Thuries, G. Cibrario, L. Brunet, F. Deprat, J.-E. Michallet, F. Clermidy, and M. Vinet. 2014. 3D sequential integration 
opportunities and technology optimization. In Proceedings of the IEEE International Interconnect Technology Conference. 373-376.

C. Bienia. 2011. Benchmarking Modern Multiprocessors. Ph.D. Diss., Princeton Univ., Princeton NJ.

N. Binkert, S. Sardashti, R. Sen, K. Sewell, M. Shoaib, N. Vaish, M. D. Hill, D. A. Wood, B. Beckmann, G. Black, S. K. Reinhardt, A. Saidi, A. Basu, J. Hestness, D. R. Hower, and T. Krishna. 2011. The gem5 simulator. SIGARCH Comput. Archit. News 39, 2 (August 2011), 1-7.

S. Bobba, A. Chakraborty, O. Thomas, P. Batude, T. Ernst, O. Faynot, D. Z. Pan, and G. De Micheli. 2011. CELONCEL: Effective design technique for 3-D monolithic integration targeting high performance integrated circuits. In Proceedings of the Asia South Pacific Design Automation Conference (ASP-DAC), 336-343.

T. Bui, C. Heigham, C. Jones, and T. Leighton. 1989. Improving the performance of the Kernighan-Lin and simulated annealing graph bisection algorithms. In Proceedings of the 26th ACM/IEEE Design Automation Conference (DAC'89). ACM, New York, 775-778.

T. Chelcea and S. M. Nowick. 2000. A low-latency FIFO for mixed-clock systems. In Proceedings of the IEEE Computer Society Workshop on VLSI System Design for a System-on-Chip Era, 119-126.

T.-C. Chen and Y.-W. Chang. 2005. Modern floorplanning based on fast simulated annealing. In Proceedings of the 2005 International Symposium on Physical design (ISPD'05). ACM, New York, 104-112.

J. Cong, J. Wei, and Y. Zhang. 2004. A thermal-driven floorplanning algorithm for 3D ICs. In Proceedings of the 2004 IEEE/ACM International Conference on Computer-Aided Design (ICCAD’04). IEEE Computer Society, Washington, DC, 306-313.

A. K. Coskun, J. L. Ayala, D. Atienza, T. S. Rosing, and Y. Leblebici. 2009. Dynamic thermal management in 3D multicore architectures. In Proceedings of the Conference on Design, Automation and Test in Europe (DATE'09). European Design and Automation Association, Belgium, 1410-1415.

A. K. Coskun, T. S. Rosing, and K. Whisnant. 2007. Temperature aware task scheduling in MPSoCs. In Proceedings of the Conference on Design, Automation and Test in Europe (DATE'07). EDA Consortium, San Jose, CA, $1659-1664$.

B. Dang, M. S. Bakir, D. C. Sekar, C. R. King, and J. D. Meindl. 2010. Integrated microfluidic cooling and interconnects for 2D and 3D chips. IEEE Trans. Adv. Packag. 33, 79-87.

S. Das, A. Chandrakasan, and R. Reif. 2004. Timing, energy, and thermal performance of three-dimensional integrated circuits. In Proceedings of the 14th ACM Great Lakes Symposium on VLSI (GLSVLSI'04). ACM, New York, 338-343.

S. Das, J. R. Doppa, P. P. Pande, and K. Chakrabarty. 2017. Monolithic 3D-enabled high performance and energy efficient network-on-chip. In Proceedings of the IEEE International Conference on Computer Design (ICCD), Boston, MA, 233-240.

S. Das, D. Lee, D. H. Kim, and P. P. Pande. 2015. Small-world network enabled energy efficient and robust 3D NOC architectures. In Proceedings of the 25th Edition on Great Lakes Symposium on VLSI (GLSVLSI'15). ACM, New York, 133-138.

K. Furumi, M. Imai, and A. Kurokawa. 2017. Cooling architectures using thermal sidewalls, interchip plates, and bottom plate for 3D ICs. In Proceedings of the 18th International Symposium on Quality Electronic Design (ISQED). 283-288.

W. Huang, S. Ghosh, S. Velusamy, K. Sankaranarayanan, K. Skadron, and M. R. Stan. 2006. HotSpot: A compact thermal modeling methodology for early-stage VLSI design. IEEE Trans. Very Large Scale Integr. Syst. 14 (2006) 501-513.

W. Huang, M. R. Stan, K. Skadron, K. Sankaranarayanan, S. Ghosh, and S. Velusam. 2004. Compact thermal modeling for temperature-aware design. In Proceedings of the 41st Annual Design Automation Conference (DAC'04). ACM, New York, 878-883.

R. Ishihara, M. R. T. Mofrad, J. Derakhshandeh, N. Golshani and C. I. M. Beenakker. 2012. Monolithic 3D-ICs with single grain Si thin film transistors. In Proceedings of the IEEE International Conference on Solid-State and Integrated Circuit Technology. 1-4.

The International Technology Roadmap for Semiconductors. 2015. ITRS Reports. Retrieved from http://www.itrs2.net/ itrs-reports.html.

W. Jang and D. Z. Pan. 2011. A voltage-frequency island aware energy optimization framework for networks-on-chip. IEEE f. Emerg. Sel. Top. Circ. Syst. 1 (2011) 420-432.

D.-C. Juan, S. Garg, and D. Marculescu. 2014. Statistical peak temperature prediction and thermal yield improvement for 3d chip multiprocessors. ACM Trans. Des. Autom. Electron. Syst. 19, 4, Article 39 (August 2014), 23 pages.

D.-C. Juan, S. Garg, J. Park, and D. Marculescu. 2013. Learning the optimal operating point for many-core systems with extended range voltage/frequency scaling. In Proceedings of the IEEE/ACM/IFIP International Conference on Hardware/Software Codesign and System Synthesis (CODES+ISSS'13). IEEE Press, Piscataway, NJ, Article 8, 10 pages.

R. G. Kim, W. Choi, Z. Chen, P. P. Pande, D. Marculescu, and R. Marculescu. 2016. Wireless NoC and dynamic VFI codesign: Energy efficiency without performance penalty. IEEE Trans. Very Large Scale Integr. Syst. 24 (2016) 2488-2501.

J. Kim, C. Nicopoulos, D. Park, R. Das, Y. Xie, V. Narayanan, M. S. Yousif, and C. R. Das. 2007. A novel dimensionallydecomposed router for on-chip communication in 3D architectures. In Proceedings of the 34th Annual International Symposium on Computer Architecture (ISCA'07). ACM, New York, 138-149. 
S. S. Kumar, A. Aggarwal, R. S. Jagtap, A. Zjajo, and R. Van Leuken. 2014a. System level methodology for interconnect aware and temperature constrained power management of 3-D MP-SOCs. IEEE Trans. Very Large Scale Integr. Syst. 22 (2014), 1606-1619.

P. Kumar, H. Yang, I. Bacivarov, and L. Thiele. 2014b. COOLIP: Simple yet effective job allocation for distributed thermallythrottled processors. In Proceedings of the Conference on Design, Automation \& Test in Europe (DATE'14). European Design and Automation Association, Belgium, Article 280, 4 pages.

J. H. Lau. 2010. TSV manufacturing yield and hidden costs for 3D IC integration. In Proceedings of the 60th Electronic Components and Technology Conference (ECTC). 1031-1042.

H. H. S. Lee and K. Chakrabarty. 2009. Test challenges for 3D integrated circuits. IEEE Des. Test Comput. 26 (2009) 26-35.

Y. J. Lee, D. Limbrick, and S. K. Lim. 2013. Power benefit study for ultra-high density transistor-level monolithic 3D ICs. In Proceedings of the 50th Annual Design Automation Conference (DAC’13). ACM, New York, Article 104, 10 pages.

S. Li, J. H. Ahn, R. D. Strong, J. B. Brockman, D. M. Tullsen, and N. P. Jouppi. 2009. McPAT: An integrated power, area, and timing modeling framework for multicore and manycore architectures. In Proceedings of the 42nd Annual IEEE/ACM International Symposium on Microarchitecture (MICRO 42). ACM, New York, 469-480.

C. Liu and S. K. Lim. 2012. A design tradeoff study with monolithic 3D integration. In Proceedings of the International Symposium on Quality Electronic Design (ISQED). IEEE, Santa Clara, CA, 529-536.

O. Lysne, T. Skeie, S. A. Reinemo, and I. Theiss. 2006. Layered routing in irregular networks. IEEE Trans. Parallel Distrib. Syst. 17 (2006), 51-65.

C. Marcon, R. Fernandes, R. Cataldo, F. Grando, T. Webber, A. Benso, and L. B. Poehls. 2014. Tiny NoC: A 3D mesh topology with router channel optimization for area and latency minimization. In Proceedings of the IEEE International Conference on VLSI Design. 228-233.

S. Murali, C. Seiculescu, L. Benini, and G. De Micheli. 2009. Synthesis of networks on chips for 3D systems on chips. In Proceedings of the Asia South Pacific Design Automation Conference (ASP-DAC). 242-247.

D. K. Nayak, S. Banna, S. K. Samal, and S. K. Lim. 2015. Power, performance, and cost comparisons of monolithic 3D ICs and TSV-based 3D ICs. In Proceedings of the IEEE SOI-3D-Subthreshold Microelectronics Technology Unified Conference (S3S). Rohnert Park, CA, 1-2.

V. Nguyen and C. Martel. 2005. Analyzing and characterizing small-world graphs. In Proceedings of the 16th Annual ACM-SIAM Symposium on Discrete Algorithms. Society for Industrial and Applied Mathematics, Philadelphia, PA, USA, 311-320.

U. Y. Ogras and R. Marculescu. 2006. It's a Small World after All: NoC performance optimization via long-range link insertion. IEEE Trans. on VLSI Systems, 14, 693-706.

U. Y. Ogras, R. Marculescu, D. Marculescu, and E. G. Jung. 2009. Design and management of voltage-frequency island partitioned networks-on-chip. IEEE Trans. Very Large Scale Integr. Syst. 17 330-341.

D. Oh, C. C. P. Chen, and Y. H. Hu. 2012. Efficient thermal simulation for 3-D IC with thermal through-silicon vias. IEEE Trans. Comput. Des. Integr. Circuits Syst. 31 (2012) 1767-1771.

S. Panth, K. Samadi, Y. Du, and S. K. Lim. 2014. Design and CAD methodologies for low power gate-level monolithic 3D ICs. In Proceedings of the 2014 International Symposium on Low Power Electronics and Design (ISLPED'14). ACM, New York, 171-176.

T. Petermann and P. D. L. Rios. 2005. Spatial small-world networks: a wiring-cost perspective, e-print arXiv:condmat/0501420.

A.-M. Rahmani, P. Liljeberg, J. Plosila, and Ha. Tenhunen. 2013. Developing a power-efficient and low-cost 3D NoC using smart GALS-based vertical channels. f. Comput. Syst. Sci. 79, 4, 440-456.

R. Rao and S. Vrudhula. 2007. Performance optimal processor throttling under thermal constraints. In Proceedings of the 2007 International Conference on Compilers, Architecture, and Synthesis for Embedded Systems (CASES'07). ACM, New York, 257-266.

M. M. Sabry, A. Sridhar, J. Meng, A. K. Coskun, and D. Atienza. 2013. Greencool: An energy-efficient liquid cooling design technique for 3-D MPSoCs via channel width modulation. IEEE Trans. Comput. Des. Integr. Circuits Syst. 32, 524-537.

S. K. Samal, D. Nayak, M. Ichihashi, S. Banna, and S. K. Lim. 2016. Monolithic 3D IC vs. TSV-based 3D IC in 14nm FinFET technology. In Proceedings of the IEEE SOI-3D-Subthreshold Microelectronics Technology Unified Conference (S3S). 1-2.

S. K. Samal, S. Panth, K. Samadi, M. Saedi, Y. Du, and S. K. Lim. 2014. Fast and accurate thermal modeling and optimization for monolithic 3D ICs. In Proceedings of the 51st Annual Design Automation Conference (DAC'14). ACM, New York, Article 206, 6 pages.

C. Santos, P. Vivet, S. Thuries, O. Billoint, J.-P. Colonna, P. Coudrain, and L. Wang. 2016. Thermal performance of CoolCube $^{\mathrm{TM}}$ monolithic and TSV-based 3D integration processes. In Proceedings of the 2016 IEEE International 3D Systems Integration Conference (3DIC). 1-5.

D. K. Schroder and J. A. Babcock. 2003. Negative bias temperature instability: Road to cross in deep submicron silicon semiconductor manufacturing. f. Appl. Phys. 94, 1-18. 
D. K. Schroder. 2007. Negative bias temperature instability: What do we understand?. Microelect. Reliab. 47, 841-852.

C. Seiculescu, S. Murali, L. Benini, and G. De Micheli. 2009. SunFloor 3D: A tool for networks on chip topology synthesis for 3D systems on chips. In Proceedings of the Conference on Design, Automation and Test in Europe (DATE'09). European Design and Automation Association, Belgium, 9-14.

C. Seiculescu, S. Murali, L. Benini, and G. De Micheli. 2010. Comparative analysis of NoCs for two-dimensional versus three-dimensional SoCs supporting multiple voltage and frequency islands. IEEE Trans. Circ. Syst. II: Express Briefs, 57, 5, 364-368.

B. Shi, A. Srivastava, and P. Wang. 2011. Non-uniform micro-channel design for stacked 3D-ICs. In Proceedings of the 48th Design Automation Conference (DAC'11). ACM, New York, 658-663.

J. C. Souriau, L. Castagné, J. L. Liotard, K. Inal, J. Mazuir, F. L. Texier, G. Fresquet, M. Varvara, N. Launay, B. Dubois, and T. Malia. 2012. 3D multi-stacking of thin dies based on TSV and micro-inserts interconnections. In Proceedings of the IEEE 62nd Electronic Components and Technology Conference. 1047-1053.

O. Turkyilmaz, G. Cibrario, O. Rozeau, P. Batude, and F. Clermidy. 2014. 3D FPGA using high-density interconnect monolithic integration. In Proceedings of the Conference on Design, Automation \& Test in Europe (DATE'14). European Design and Automation Association, Belgium, Article 338, 4 pages.

T. Uhrmann, T. Wagenleitner, T. Glinsner, M. Wimplinger, and P. Lindner. 2014. Monolithic IC integration key alignment aspects for high process yield. In Proeedings of the 2014 SOI-3D-Subthreshold Microelectronics Technology Unified Conference (S3S). 1-2.

N. Watanabe, H. Shimamoto, K. Kikuchi, M. Aoyagi, H. Kikuchi, A. Yanagisawa, and A. Nakamura. 2016. Wet cleaning process for high-yield via-last TSV formation. In Proceedings of the IEEE International 3D Systems Integration Conference (3DIC). 1-4.

N. Watanabe, H. Kikuchi, A. Yanagisawa, H. Shimamoto, K. Kikuchi, M. Aoyagi, and A. Nakamura. 2017. Development of a high-yield via-last through silicon via process using notchless silicon etching and wet cleaning of the first metal layer. Japan, 7. Appl. Phys., 56.

S. C. Woo, M. Ohara, E. Torrie, J. P. Singh, and A. Gupta. 1995. The SPLASH-2 programs: Characterization and methodological considerations. In Proceedings of the 22nd Annual International Symposium on Computer Architecture (ISCA'95) ACM, New York, 24-36.

Y. Xu, Y. Du, B. Zhao, X. Zhou, Y. Zhang, and J. Yang. 2009. A low-radix and low-diameter 3D interconnection network design. In Proceedings of the International Symposium on High-Performance Computer Architecture. 30-41.

P. M. Yaghini, A. Eghbal, S. S. Yazdi, and N. Bagherzadeh. 2015. Accurate system-level TSV-to-TSV capacitive coupling fault model for 3D-NoC. In Proceedings of the 9th International Symposium on Networks-on-Chip (NOCS'15). ACM, New York, Article 3, 8 pages.

P. Zhou, P. H. Yuh, and S. S. Sapatnekar. 2010. Application-specific 3D network-on-chip design using simulated allocation. In Proceedings of the Asia South Pacific Design Automation Conference (ASP-DAC). 517-522.

Received January 2018; revised April 2018; accepted May 2018 